\title{
TURBULENCE, MAGNETISM, AND TRANSPORT INSIDE STARS
}

\author{
A.S. Brun ${ }^{1}$ and A. Strugarek ${ }^{1}$
}

\begin{abstract}
We present recent progress made in modelling stars and their turbulent magnetized dynamics in 3-D. This work is inspired by many years of discussion with Jean-Paul Zahn. I (ASB) first met him as a professor of astrophysical fluid dynamics (AFD) at the Paris-Meudon observatory's graduate school of astrophysics in 1994-1995. He made me the honor of accepting to be my PhD's advisor (1995-1998). He then supported me during my postdoc years in Boulder with his long time friend Prof. Juri Toomre between January 1999 and December 2002 and through the difficult process of getting a tenure position, and then since as a tenure researcher in Department of Astrophysics at CEA Paris-Saclay. I have been fortunate and lucky to share so many years discussing and doing scientific projects with Jean-Paul. As I was getting more experienced and started supervising my own students, he was always available, guiding us with his acute scientific vista and encouraging them. Antoine Strugarek, who co-author this paper, was like me fortunate to share Jean-Paul's knowledge. The three of us published several papers together during Antoine's PhD (2009-2012) addressing the dynamics of the solar tachocline and its interplay with convection. We miss him greatly. In this paper, we discuss mainly two topics that benefited from Jean-Paul's deep understanding of AFD: a) the dynamics of the solar tachocline and angular momentum transport in stellar interior and b) turbulent convection and dynamo action in stellar convection zones.
\end{abstract}

\section{Introduction}

Solar-like stars possess two main zones: a deep stably stratified radiative interior hosting the nuclear core and an extended unstable stratified turbulent convective envelope. Understanding the magnetohydrodynamics (MHD) of each zone is a challenge by itself, so their non linear and thermodynamic coupling even more

${ }^{1}$ Department of Astrophysics \& UMR AIM, CEA Paris-Saclay, CNRS/INSU, University of Paris 7, Gif-sur-Yvette, France 
so! Jean-Paul's pivotal work on these various topics is at the fundation of our theoretical and numerical developments on these topics of stellar fluid dynamics (SFD). Some of the key elements that one must explain in SFD are the following:

- How do the large scale mean flows (differential rotation and meridional circulation) observed at the solar surface arise and what explains their peculiar profiles as a function of depth and latitude? For instance why is the solar differential rotation inverted by helioseismic techniques conical at midlatitudes, and not cylindrical as originally anticipated by applying rotating fluid dynamics and Taylor-Proudman constraints? Why is there a transition to uniform body rotation in the radiative zone, the so-called tachocline (neologism created by Jean-Paul and Ed Spiegel in their seminal 1992 paper), and what makes it so shallow (less than $4 \%$ of the solar radius)? How many cells in latitude and radius does the meridional circulation possess? Do the number of cells vary with the solar cycle?

- What sets the period of the solar cycle to 11 yr? Typical convection time scales are of the order of minutes to months not years, so the solar magnetic cycle period corresponds to about 130 times the slowest dynamical time scale of the convective envelope. Viscous diffusion time scale is very very slow (beyond billion years) in the quasi inviscid Sun as does any time scale based on diffusive processes (thermal, magnetic, compositional) computed with atomic values.

- How intense are penetrative convection and overshooting at the base of the convection zone? What are the intensity and characteristic scales of convective plumes? What amplitude of gravity waves are being excited by these pummeling down flows and how are these waves propagating and depositing angular momentum, if any, in the radiative interior?

- Is there a fossil field inside the radiative core and if so what is its amplitude and geometry? Can it be detected through its influence on the solar surface magnetic field?

- What is the rotation profile deep inside the radiative zone? How well mixed are chemicals there?

Jean-Paul's seminal work in the papers published in late 80's/early 90's (and in the following next two decades), have adressed many of these questions and proposed original ideas to resolve them. We will present them along with non linear 3-D MHD simulations that have often confirmed these ideas. In Section 2 we discuss the dynamics and confinement of the solar tachocline, in Section 3 we discuss solar-like star magnetism and dynamo action. In Section 4 we reflect upon our findings and conclude. In Section 5 we reproduce the texte one of us (A.S. Brun) wrote in Jean-Paul's memory for the Père-Lachaise ceremony of the 1st of August 2015 . 


\section{Solar tachocline}

The solar tachocline (Spiegel \& Zahn 1992) was initially identified as a transition region in the internal differential rotation profile of the Sun (Brown et al. 1989). Thanks to helioseismology (e.g. Schou et al. 1998; Thompson et al. 2003), it was quickly realized that the internal rotation profile of the Sun is constant in the radiative interior ${ }^{1}$, and non uniform (differential) in both radius and latitude in the convective envelope. A shear layer necessarily separates the two, and was called tachocline. Helioseismic inversions were able to deduce that the thickness of the tachocline is less than $4 \%$ of the solar radius (e.g. Kosovichev 1996; Basu 1997; Corbard et al. 1999; Elliott \& Gough 1999; Charbonneau et al. 1999a; Basu \& Antia 2003). Due to its strong velocity shear, the tachocline is often invoked in dynamo models as a region were strong toroidal fields can be generated (through shear) and/or stored (the bulk of the tachocline lies at the top of the radiative region, were the turbulent motions only arise from either local magnetohydrodynamic instabilities or the penetration of convective motions from above). Note though that the importance of a tachocline for solar-type dynamos is currently debated in the community (e.g. Wright \& Drake 2016, and Sect. 3).

We give a look back here at the original work of Spiegel \& Zahn (1992) that first theorized the long-term evolution of the tachocline, which lead to the tachocline confinement problem. More than 25 years later, this problem is still a matter of lively debates in the solar physics community, showing the important impact of this pioneering work of Jean-Paul and Ed Spiegel. We review in this section the various scenarii that have been proposed to confine the tachocline to its observed thickness.

\subsection{Setting up the scene: The radiative spreading of the tachocline}

Spiegel \& Zahn (1992) showed that the solar tachocline (as we know it presently) should slowly diffuse into the radiation zone on a hyper-diffusive Eddington-Sweet time scale defined as

$$
\tau_{\mathrm{ES}}=\left(\frac{N}{2 \Omega}\right)^{2} \tau_{\kappa},
$$

where $N$ is the Brünt-Vaisälä frequency, $\Omega$ the rotation rate of the star, and $\tau_{\kappa}=R^{2} / \kappa$ the radiative diffusion time-scale $(\kappa$ is the radiative diffusivity, and $R$ the typical length-scale over which the diffusion occurs). The mechanism they highlighted proceeds as follows:

- Convective motions under the influence of rotation sustain non-linearly a differential rotation, primarily in latitude in the convective envelope (see Fig. 1).

- This latitudinal shear permeates the top of the tachocline.

\footnotetext{
${ }^{1}$ In the core (below $0.2 \mathrm{R}$ ), rotation possibly increases again, see Garcia et al. (2007), Fossat et al. (2017).
} 

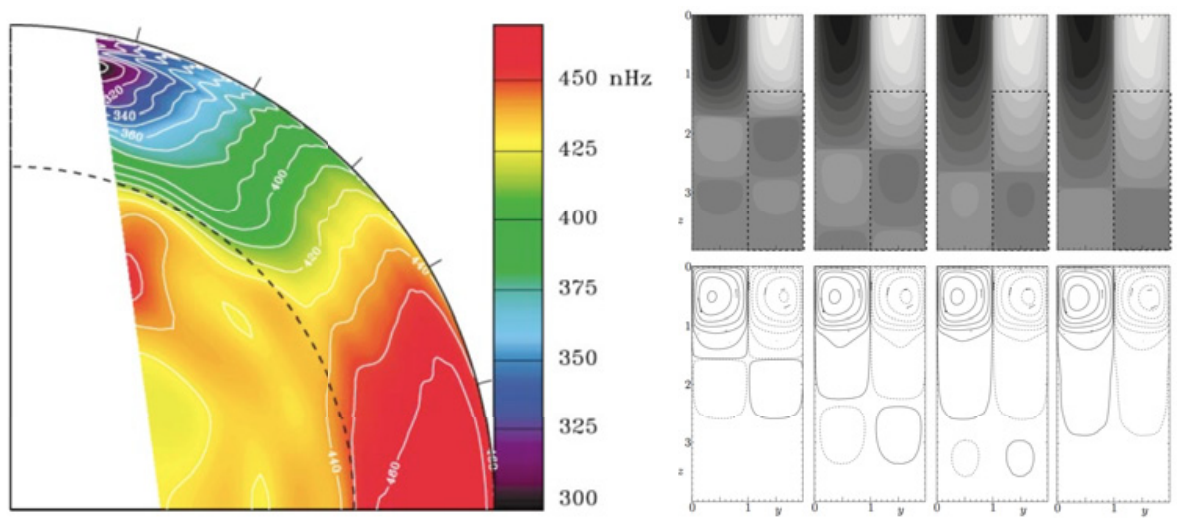

Fig. 1. Left: Differential rotation of the solar interior observed with helioseismology (Schou et al. 1998; Thompson et al. 2003) Right: Numerical model of the radiative spreading of the differential rotation into the radiative core (top) along with the meridional circulation burrowing (bottom) (Wood \& Brummell 2012).

- A meridional circulation also naturally emerges there, either through gyroscopic pumping from the differential rotation profile (McIntyre 2007), or from a Vogt-Eddington mechanism (see, e.g. Wood et al. 2002).

- The meridional circulation transports heat from the hot pole to the cool equator, and this transport is balanced by radiative thermal dissipation that ultimately determines the strength of the meridional flow, due to the aforementioned balance.

- The meridional circulation then burrows downwards on an Eddington-Sweet time-scale (Eq. (2.1)).

- Because meridional flows transport angular momentum, the differential rotation profile spreads inward as well, leading to the so-called radiative spreading phenomenon (Spiegel \& Zahn 1992).

Starting with a sharp shear transition at the base of the convection zone, Spiegel \& Zahn (1992) showed it should have spread over 30\% of the solar radius by now (see also Wood \& Brummell 2012, for a detailed parametric study of this radiative spreading and the right panel of Fig. 1). Hence, one has to come up with a way to confine the solar tachocline to its observe thickness, in addition to a way of maintaining an almost solid-body rotation in the radiative interior.

\subsection{Tachocline confinement scenarii}

Several theories for the confinement of the tachocline have emerged since Spiegel \& Zahn (1992), which we review here. Some of them focus only on the confinement problem itself, while others also offer an explanation for the solid-body rotation of the internal radiative zone. We decompose the different theories in two classes: the 
slow and the fast tachocline (Gilman 2000), which rely on a confinement that establishes respectively on a secular or dynamical timescale.

\subsubsection{Slow tachocline theory}

One way to confine the tachocline is to rely on the existence of an inner magnetic field. Interestingly, a large-scale axisymmetric magnetic field in the radiation zone could in principle also explain why it rotates roughly like a solid body (Rüdiger \& Kitchatinov 1997). This can be achieved through the homogenization of the rotational profile along the magnetic field lines (the so-called Ferraro's law of iso-rotation Ferraro 1937). Hence, many studies were aimed at exploring the possible existence of such a hypothetical magnetic field and its stability and dynamical evolution, to assess if it could explain the observed solar inner rotation profile (Mestel et al. 1988; Mestel \& Weiss 1987).

Despite the appeal of the magnetic scenario to explain the solar internal rotation profile, it has to be noted that an organized large-scale magnetic field does not remain naturally confined and tends to diffuse out on ohmic diffusion timescales. The ohmic diffusion time $\tau_{\eta} \sim R^{2} / \eta$ (where $R$ is the characteristic length over which the field diffuses and $\eta$ is the ohmic diffusion coefficient) is long in the solar radiation zone $(\sim \mathrm{Gyr})$. Nevertheless, at the age of the Sun a significant part of such magnetic field would have diffused out and encountered the tachocline (and eventually the convection zone above), which could severely affect both the topology of the magnetic field and its effect on the rotation profile. Gough \& McIntyre (1998) (noted GM98 hereafter) proposed a scenario in which the interior magnetic field could be confined by a penetrating meridional flow coming from the convection zone. A significant amount of work has been carried out since to assess the reality of this scenario non-linearly, with the help of numerical simulations. The first numerical attempt to model the GM98 scenario was carried out by Garaud (2002) in 2D. Taking into account the radiation zone only, she showed that the magnetic field lines should open into the tachocline and transport angular momentum throughout the solar interior. Similar results were obtained by Brun \& Zahn (2006) in 3D. They also observed the development of magnetic instabilities, confirming that a purely axisymmetric dipolar field was unstable in 3D and could not be a likely candidate for the tachocline confinement scenario. In order to better test the GM98 scenario, ad-hoc meridional circulations (supposedly originating from the unresolved convection zone) were added to the numerical models (Sule et al. 2005; Rüdiger \& Kitchatinov 2007; Garaud \& Garaud 2008). They confirmed that in order for the GM98 scenario to be valid the amplitude and shape of the meridional circulation must be chosen with care otherwise the tachocline spreads. The meridional flow penetration at the base of the convection zone is also an important ingredient of that model but it is currently poorly constrained by observations, as part of the field seems to always be able to connect to the upper convection zone. In order to test the GM98 scenario more self-consistently, two paths have been recently followed.

First, a better understanding of the meridional circulation penetration was developed in reduced models by Garaud \& Brummell (2008), Garaud \& 

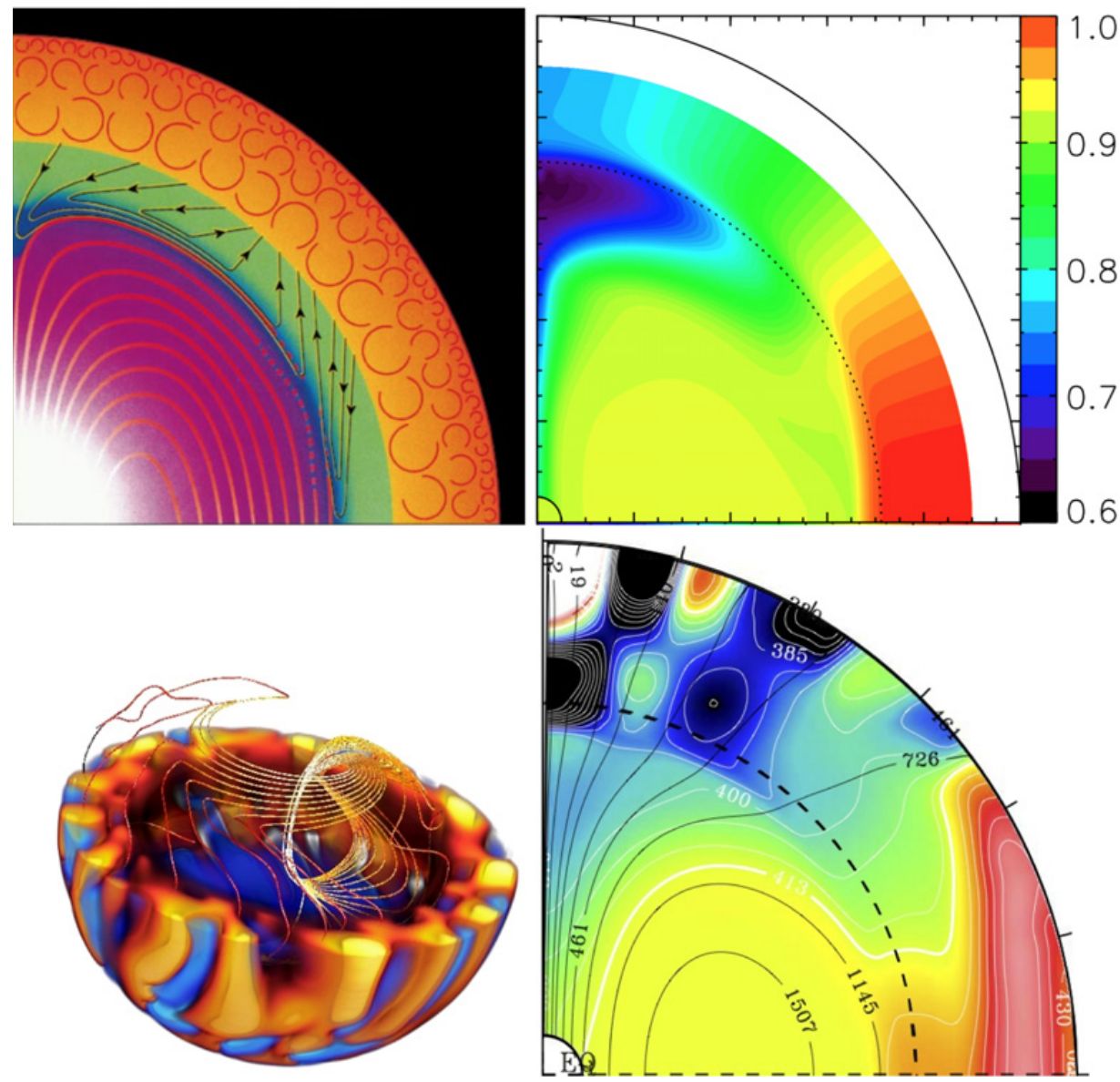

Fig. 2. Top Left: Schematics of the GM98 scenario of a slow tachocline (Gough \& McIntyre 1998). Top Right: 2D simulations attempt (unsuccessfully) to realize the GM98 scenario by Acevedo-Arreguin et al. (2013). Bottom Right: Likewise 3D simulation confirming the difficulty to realize the GM98 scenario by Strugarek et al. (2011a). Bottom Left: 3D view of the same simulation showing the magnetic coupling between the internal radiative zone and the convective envelope leading to the failure of the magnetic confinement scenario.

Acevedo Arreguin (2009), Wood \& Brummell (2012). These studies shed light on the importance of the parameter $\sigma=\frac{N}{2 \Omega} \sqrt{\operatorname{Pr}}$ (where $\operatorname{Pr}=\nu / \kappa$ the Prandtl number). They identified various regimes of penetration of meridional flows depending on the value of $\sigma$ at the top of the tachocline and on the boundary conditions of the model. In the Sun, this parameter is much lower than 1 , which is a difficult regime (very low Prandtl number) to achieve in numerical simulations. 
Second, global models coupling a convection zone to a radiation zone were recently developed by Rogers (2011), Wood \& Garaud (2011), Acevedo-Arreguin et al. (2013) in 2D and by Brun et al. (2011), Strugarek et al. (2011a) in 3D (see Fig. 2). A self-consistent tachocline radiatively spreading in time naturally develops in those models. Studying the GM98 scenario in a cartesian geometry where they force large scale flows (mimicking the convection without taking into account the convective motions), Wood \& Garaud (2011) showed they could obtain the magnetic confinement of the tachocline for values of $\sigma$ in a certain limited range. Acevedo-Arreguin et al. (2013) performed 2D spherical axisymmetric numerical simulations and explored a large range of the $\sigma$ parameter. They obtained various steady-state solutions in which the internal rotation profile varies significantly. However, none of their solutions fully recover the close-to solid body rotation for the radiation zone constrained by helioseismology observations. As also noted by Braithwaite \& Spruit (2017), this failure of the confinement scenario is linked to the inevitable coupling of the buried magnetic field to the convective enveloppe, either due to the entrainment by the meridional flow or convective motions themselves. Both Rogers (2011) and Strugarek et al. (2011a) also report the lack of magnetic field confinement inside the radiative zone when the convection zone flows (both large-scale means flows and small-scale turbulent flows) are modeled self-consistently. It is worth noting that Rogers (2011) reports a tachocline confinement without magnetic field, though the tachocline may still be slowly thickening due to viscous spreading in her simulations. The axisymmetric magnetic field considered by Strugarek et al. (2011a) was shown to pervade through the tachocline into the convection zone and to easily transport angular momentum between the two zones. Working closely with Jean-Paul, who always showed a communicative enthusiasm to explore new ideas, we also tested whether other magnetic configurations could also lead to the coupling of the two zones. In Strugarek et al. (2011b) we considered the case of tilted magnetic dipoles, up to the singular case of the perpendicular dipole (pure $m=1$ dipole, Strugarek, private communications). In all cases, we obtained that the magnetic field was able to pervade the tachocline and that both Maxwell torques and stresses transported angular momentum in such a way that the final rotation profile could not be reconciled with observations. The possibility to confine the tachocline through this mechanism is thus still debated today. Dynamical three dimensional numerical simulations, treating the coupling between convective and radiative regions, still need to be carried out in the correct $\sigma<1$ parameter regime, using global spherical geometry. This is a demanding regime, as it requires a fairly low Prandtl number in a strongly stratified medium.

While this scenario has arguably received a lot of attention, other alternative scenarii have also emerged in the literature to account for the thinness of the tachocline.

\subsubsection{Fast tachocline theories}

We describe here scenarii for the confinement of the tachocline based on fast, dynamical time-scales (Gilman 2000), compared to the typical Eddington-Sweet time-scale on which the slow tachocline theory relies. 
The tachocline pervades the base of the convection zone down to the upper part of the stably stratified radiative core. Several physical processes can lead to MHD turbulence in this region. Depending on the properties of this turbulence, a net transport of angular momentum can occur, either reinforcing the differential rotation imposed from the above convection zone (and thus accelerating the radiative spreading process), or opposing it up to a point where the burrowing could be stopped. The latter hypothesis based on a strongly anisotropic turbulent viscosity was first proposed by Spiegel \& Zahn (1992). They evaluated the minimum horizontal dissipation required to halt the radiative spreading of the tachocline and found that such a confinement could be realized for typical turbulent viscous coefficients. However, it was argued (Gough \& McIntyre 1998) that such 2D turbulence would likely strengthen the latitudinal gradient of the differential rotation rather than opposing it, and that this confinement theory was subsequently not realistic. The direction of momentum transport by turbulence in the tachocline is nevertheless far from being understood, due to the presence of both a strong stratification, a large-scale sheared-flow in both the latitudinal and radial direction, and the inevitable presence of magnetic fields.

But first, where does such a turbulence come from? One likely possibility comes from the convective flows above the tachocline. The strongly concentrated downflows penetrate in the upper part of the tachocline, constantly hammering the radiative zone and generating gravity waves see (Sect. 3 and Alvan et al. 2014a, 2015a). The exact nature of this mechanism, being either overshooting (the downflows slightly over-extend in the stable layer below) or penetration (the penetrating downflows locally change the properties of the background equilibrium Zahn 1991), is currently in debate in the community. The most resolved calculations of this phenomenon were recently carried out by Hotta (2017), which predict that the convective overshooting will occur on a very thin layer of about $250 \mathrm{~km}(0.03 \%$ of the solar radius). In any case, the penetrating plumes will excite some turbulence on a range of spatio-temporal scales, at least in the upper part of the tachocline.

The response of an hydrodynamical, sheared, stably-stratified layer to such excitation by overshooting turbulence was studied analytically Leprovost \& Kim (2006), Kim \& Leprovost (2007), Leprovost \& Kim (2009) and numerically by Miesch $(2001,2003)$. In all studies, the turbulent transport of angular momentum in radius and latitude was shown to strongly depend on the assumptions of the model: what are the properties of the turbulence forcing (vortical or not), what large-scale shear is considered? Both diffusive and anti-diffusive transports were observed depending on these assumptions. In the most realistic case, i.e. a strongly-stratified layer sheared in radius and latitude and forced with a vortical spectrum, Miesch (2003) showed that the induced turbulence in the tachocline would transport angular momentum diffusively in the latitudinal direction and anti-diffusively in the radial direction. Such turbulent behaviour would help confining the tachocline against radiative spreading, in a slightly diffrent spirit than the original anisotropic turbulence scenario first proposed by Spiegel \& Zahn (1992). It must finally be noted that these studies completely ignored the magnetic field that is likely to pervade the upper part of the tachocline at least 
because of the dynamo operating in the convective enveloppe above (see Sect. 3 and Browning et al. 2006). In a reduced model, Tobias et al. (2007) showed that the turbulent transport in the upper tachocline in presence of a magnetic field is neither diffusive nor anti-diffusive. While this conclusion has not been demonstrated to hold for various types of turbulent forcing, it certainly questions the realism of purely hydrodynamical scenarii for the confinement of the tachocline.

In addition to the convective forcing, some turbulence (or at least some small scale fluctuations transporting angular momentum) can be triggered due to instabilities of the large scale latitudinal and radial shear of the differential rotation, and/or the large-scale azimuthal magnetic field in the tachocline. Gilman \& Fox (1997) were the first to show that the latitudinal shear in the solar tachocline, which was shown to be hydrodynamically stable (Watson 1981; Charbonneau et al. 1999b), could be unstable in the presence of a toroidal magnetic field. After these pioneering works, HD and MHD instabilities have been extensively studied by various groups in the context of the tachocline (to cite only a few, see Cally et al. 2003; Miesch 2007; Dikpati et al. 2009; Dikpati 2012; Lawson et al. 2015; Gilman 2017, and references therein, see also the top panels of Fig. 3). The complexity of taking into account both radial and latitudinal shears in the presence of localized magnetic fields in the extreme parameters of the solar interior is still ahead of us as of today. Such instabilities, though, provide another interesting source of turbulent angular momentum transport that could play a role in the confinement of the tachocline (see discussion above).

Finally, an alternative magnetic scenario involves the 11-yr cyclic variations of the solar magnetic field and the associated feedback of the Lorentz force on the differential rotation. By quenching the latitudinal shear at the base of the convection zone, such a field could alleviate the problem of the radiative spreading of the tachocline (Forgács-Dajka \& Petrovay 2002). The strength of this scenario lies in the fact that we know the Sun possesses a cyclic large-scale magnetic field. Based on our understanding of magnetized penetrative convection (Tobias et al. 2001; Browning et al. 2006), this magnetic field should penetrate at least in the upper part of the solar tachocline. On one hand, the cyclic magnetic field changes polarity over 11 years and is sustained by turbulent convective motions which characteristic timescale is of the order of a month (see Sect. 3). On the other hand, the solar tachocline has to be confined over a timescale of $\sim 10^{6}$ years. This renders the design of a self-consistent 3D MHD model of a dynamo-confined tachocline very challenging, to say the least.

As a result, simplified models with a prescribed cyclic magnetic field were designed to test this scenario. Forgács-Dajka \& Petrovay (2001) were arguably the first pushing forward this idea. They considered a very simple model of a fast tachocline with a magnetic Prandtl number of 1 , an oscillatory toroidal field imposed from the above convection zone penetrating into the stable interior (see Garaud 1999; Dikpati et al. 2006), and a prescribed poloidal field throughout the domain. They found that if the turbulent ohmic dissipation was able to reach values of $\eta \sim 10^{10} \mathrm{~cm}^{2} / \mathrm{s}$, a kilogauss cyclic magnetic field penetrating the top of the tachocline was able to confine it against turbulent viscous dissipation. Theses 

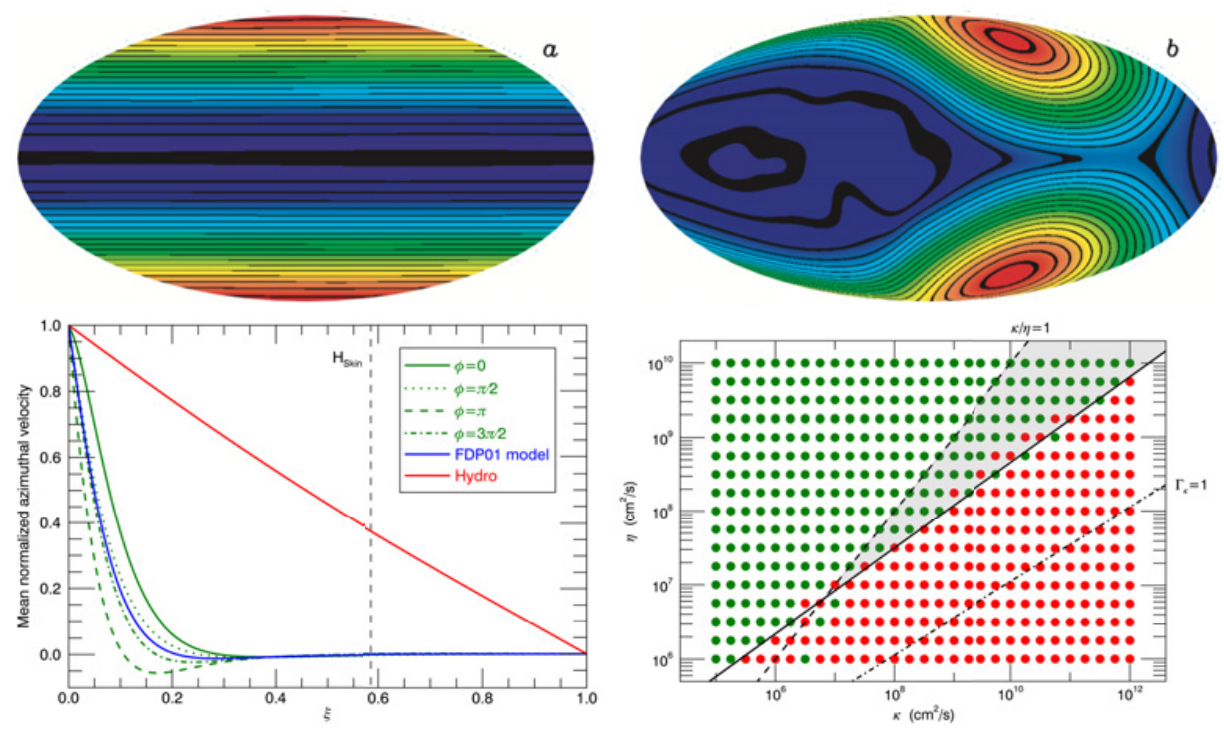

Fig. 3. Top: Clam-shell instability of the magnetic field in the tachocline (Gilman et al. 2007). Bottom left: Confinement of the tachocline with the cyclic dynamo field with the model of Forgács-Dajka \& Petrovay (2001) (Barnabé et al. 2017). Bottom right: Domain of application of the dynamo confinement scenario of the tachocline, as a function of the viscous $(\nu)$ and thermal $(\kappa)$ dissipation coefficients (the confinement occurs for the green circles, and the shaded area corresponds to realistic parameters for the tachocline, see Barnabé et al. 2017).

results were further confirmed in subsequent works (Forgács-Dajka \& Petrovay 2002; Forgács-Dajka 2004) where the authors alleviated several crucial assumptions (poloidal field evolution consistent with the toroidal field, the influence of meridional circulation, and different magnetic Prandtl numbers). This scenario was further shown to be compatible with standard flux-transport dynamo model of the Sun by Karak \& Petrovay (2013). Recently, Barnabé et al. (2017) ${ }^{2}$ revisited this scenario following some of Jean-Paul Zahn's unpublished work, where a dephasing between the poloidal and toroidal components of the field was shown to have only a mild impact on this confinement scenario. They also tested the possibility to confine the tachocline over longer timescales against radiative spreading (supposing that the turbulence in tachocline may not transport effectively angular momentum, see discussions above). They concluded that the fast tachocline confinement scenario with a cyclic dynamo field is robust as long as the turbulent ohmic dissipation in the tachocline reaches values of the order $\eta \sim 10^{8} \mathrm{~cm}^{2} / \mathrm{s}$. Ultimately, self-consistent models of the cyclic solar dynamo including a tachocline

\footnotetext{
${ }^{2}$ Jean-Paul co-authored this work and sadly passed away while we were finishing the study.
} 
are now needed to assess the realism of this scenario, that was explored only with very simplified models up to now (see Sect. 3.3).

\section{Dynamo action in stars: Role of turbulent convection and large scale flows}

Most stars possess extended convection layers, either at their surface or deep inside their core. Understanding the redistribution of heat, energy and angular momentum in these turbulent convection zones is crucial and has a direct impact on their short and long term evolution. Moreover all stars rotate, from very slow red giant (RGB) stars up to rapidly rotating young T-Tauri's, and it is equally important to assess their rotation profile and associated mixing properties.

Jean-Paul Zahn has played a pivotal role in our understanding of stellar convection, rotation and the transport of angular momentum and mixing of chemical species they incur in seminal papers such as Zahn (1983, 1991, 1992); Spiegel \& Zahn (1992) and many others. The development of high performance numerical simulations in 2-D and then in 3-D to characterize the complex stellar turbulent dynamics has always been encouraged by him, even if his mathematical skills for analytical works did not needed them per se. It was always a great pleasure to discuss with him the outcome of these simulations and to use diagnostic equations to better interpret them, as we will discuss in Section 3.2.

Along with purely hydrodynamical processes such as convection or rotation, another key ingredient influences the internal and surface dynamics of stars, e.g. magnetic field. Indeed, the Sun is a magnetic star, with a clear 11-yr sunspot cycle and complex surface magnetism. Moreover it is far from being the only one in the Universe. Clear evidence for stellar magnetic activity have been collected by various observational programs and satellites over the last 50 years. In particular, regular activity cycles have been reported in recent years (Baliunas et al. 1995; Saar \& Brandenburg 1999; Saar 2002; Egeland et al. 2017). Other stars such as Cp (Ap \& Bp) stars (Donati \& Landstreet 2009) do not show cycles but instead a strong and oblique magnetic field in their radiative envelope leading to a modulated signal (Aurière et al. 2007). One characteristic of stellar magnetism that is key to note is that stars with a spectral type later than $\mathrm{F}$ generally exhibit strong and time dependent magnetic signals such as revealed by X-ray proxies (Pizzolato et al. 2003; Wright et al. 2011), CaII H-K lines proxies (Hall et al. 2009) or more recently frequency shifts of acoustic modes using asteroseismic techniques (see Garcia et al. 2010; Brun et al. 2015a; for a recent review). On the contrary more massive stars, such as A or B stars show much less systematically the presence of magnetic field $(<10 \%)$. There are thus evidence that the presence of intense and variable magnetism in stars is related to the location of their convection zone and the existence or not of surface convection dynamo. Convection and its associated mean flows are thus expected to play a central role in our attempt to model and understand stellar magnetism. Of course stars are complex objects and these simple considerations can only be used as a guide to develop more realistic models of stars and of their turbulent convection and magnetism. In the next subsections we 
will briefly discuss recent theoretical and numerical studies that have attempted to model and to understand the complex interplay between convection, rotation and magnetic fields in stars (see the recent review by Brun \& Browning 2017, for more in depth discussion of the solar-stellar connection). This work has benefited from many years of joint discussions with Jean-Paul and Juri and comes in the direct continuation of their joint publication on stellar convection and penetration (Latour et al. 1981; Hurlburt et al. 1994; see also Juri Toomre's contribution in these proceedings).

\subsection{Numerical simulations as an efficient tool for studying stellar MHD}

Thanks to the development of massively parallel computers, direct 3-D nonlinear numerical simulations of stellar magnetohydrodynamics has been undertaken over the last 30 years. Two approaches have been pursued: local high resolution simulations that describe the small scale turbulent motions and local dynamo (Cattaneo 1999; Vögler \& Schüssler 2007; Stein 2012) and global ones that take into account the correct geometry and the existence of large scale flows in rotating convective zones (Rubin et al. 1985; Glatzmaier 1985; Brun et al. 2004; Miesch \& Toomre 2009; Racine et al. 2011; Käpylä et al. 2012; Hotta et al. 2016; Brun et al. 2017; Strugarek et al. 2017). One of Jean-Paul's interest was to understand global properties of stars. He helped and encouraged the community in developing global models of stellar convection and dynamos. These simulations allow us to describe turbulent, rotating and magnetized convection either in a full sphere, a shell or a wedge-like geometry ( $c f$. the recent book by Charbonneau 2013). Contrary to the kinematic mean field stellar dynamo approach, 3-D simulations use the full set of MHD equations ${ }^{3}$. These equations couple classical fluid mechanic equations to the induction equation and as such do not prescribe the velocity field (convection and mean flows) but compute it self consistently (cf. Brun et al. 2004; Miesch 2005). This is the main advantage of the global full MHD approach: the influence of rotation and magnetic field on turbulence and 3D convection is computed nonlinearly, not parametrized. We refer the reader to the extensive review of stellar magnetism recently published in by Brun \& Browning (2017).

Over the last three decades several groups have developed stellar convection and dynamo models in spherical geometry following the pioneering work of P. Gilman and G. Glatzmaier in the early-mid 80's (Gilman \& Miller 1981; Glatzmaier \& Gilman 1982; Gilman 1983; Glatzmaier 1985). Note that Jean-Paul is being acknowledged in the first article of Gilman and Glatzmaier for having proposed a solution for improving their models of solar global convection, demonstrating if needed its keen interest for these $3 \mathrm{D}$ simulations ${ }^{4}$.

\footnotetext{
${ }^{3}$ We refer to Küker \& Rüdiger (2008), Hotta \& Yokoyama (2011), Kitchatinov \& Olemskoy (2012); for simpler 2-D models of stellar differential rotation assuming a parametrization of turbulent convection.

${ }^{4}$ Note that Jean-Paul was also one of the founders of CERFACS in Toulouse.
} 
Prominent examples of modern global or wedge-like parallel codes used to carry original studies in recent years on stellar convection and dynamo include:

- the Anelastic Spherical Harmonics (ASH) code: Miesch et al. (2000); Brun \& Toomre (2002), Ballot et al. (2007), Miesch et al. (2008), Browning (2008), Bessolaz \& Brun (2011), Augustson et al. (2012), Brun et al. (2017) on surface convection and zonal flows, Browning et al. (2004), Alvan et al. (2014b, $2015 b)$ on gravity waves generation and propagation in stellar radiative interior and Brun (2004), Brun et al. (2004), Browning et al. (2006), Brown et al. (2008), Browning 2008; Jouve \& Brun 2009; Brown et al. (2010, 2011), Brun et al. (2011), Miesch \& Brown (2012), Nelson et al. (2013), Augustson et al. (2013), Jouve et al. (2013), Pinto \& Brun (2013), Nelson et al. (2014), Augustson et al. (2015), Varela et al. (2016) on solar-like star dynamo action, cycles, creation of large scale fibril magnetic wreaths and flux emergence (see also the Rayleigh code - N. Featherstone),

- the Eulag MHD code: Ghizaru et al. (2010), Racine et al. (2011), Charbonneau (2013), Smolarkiewicz \& Charbonneau (2013), Lawson et al. (2015), Strugarek et al. (2017) on solar and stellar dynamo and cycles,

- the PENCIL code: Brandenburg \& Dobler (2002), Mitra et al. (2009), Warnecke et al. (2013), Käpylä et al. (2013), Guerrero et al. (2013), Warnecke et al. (2014), Karak et al. (2015), Käpylä et al. (2016), Warnecke et al. (2016), Käpylä et al. (2017) on stellar convection, dynamos and cycles,

- the Magic code: Wicht (2002), Christensen \& Aubert (2006), Christensen et al. (2009), Christensen (2010), Gastine et al. (2013), Gastine et al. (2014), Yadav et al. (2015), Jouve et al. (2015), Wicht et al. (2017) on convection, dynamos in stars and magnetic instabilities in radiative interior,

- Several research groups in Japan: Takehiro \& Hayashi (1999), Yoshida \& Kageyama (2004), Kimura et al. (2011) on convection or zonal flows or Kageyama \& Sato (1997), Nishikawa \& Kusano (2008), Masada et al. (2013), Hotta et al. (2015), Hotta et al. (2016) on dynamo, solar convection and magnetism, the most recent studies making use of the "ying-yang" grid to map a spherical shell (Kageyama \& Sato 2004).

Other codes have been mostly used to model the geodynamo with some applications to the dynamo in M dwarfs (see Brun et al. 2015b for a detailed list).

Many of the codes used in the geophysics community were originally developed to model the geodynamo. They therefore rely on the Boussinesq approximation, which is not appropriate for highly stratified and compressible convective envelope as found in solar-like stars. Scientists in charge of the development of these parallel codes have converted them to be able to deal with stellar-like stratified flow by using the anelastic approximation (Gough 1969; Glatzmaier 1984; Vasil et al. 2013) and have validated them with a recent international benchmark (Jones et al. 

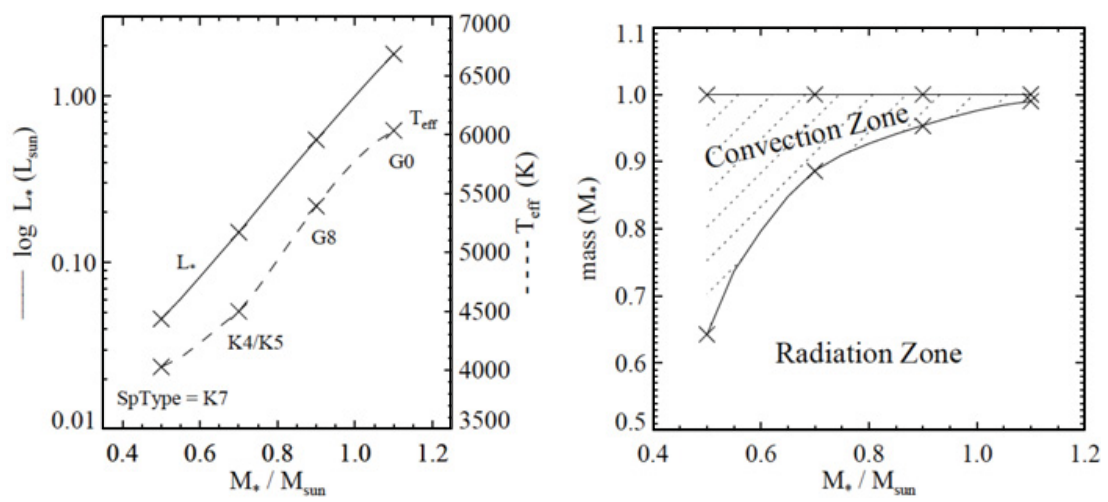

Fig. 4. Stellar luminosity, effective temperature and mass contained in the convective envelope vs. spectral type in solar like stars at solar metallicity (computed with the CESAM code Morel 1997).

2011). Direct detailed comparison between stellar 3-D MHD codes such as ASH and Eulag MHD has also been undertaken and holds very good agreements despite the very different numerical schemes used (Strugarek et al. 2016).

As we can see over the last couple of decades or so, a wealth of 3-D advanced numerical simulations and the associated high performance parallel codes have been produced. There is now a general consensus emerging on the nature of the flow and field that stellar convective envelope can produce and how sensitive they are to parameter changes. In the next sub-sections we will briefly summarize these results with some emphasis on the establishment and nature of the large scale mean flows and on the building of cyclic magnetic field through complex dynamo action.

\subsection{Stellar convection and large scale flows}

Convection in stars can variously be found in cores (massive stars), deep envelopes (solar-like stars or evolved stars), or occupy the whole star (young pre-main sequence stars or very low mass stars, with spectral type M3 or later). In this brief review, we focus our discussion on solar-like stars possessing a deep convective envelope. For a recent extended review of all type of main sequence stars we refer the reader to Brun \& Browning (2017), and references therein.

Convection plays a key role in transporting the star's heat by having strong correlations between vertical motions and temperature fluctuations (e.g. hot, low density fluid parcels go up). It is an intrinsically turbulent process as the Rayleigh number ( $R a$, e.g. the ratio between buoyancy driving and dissipation process) is huge in stars, of order $10^{18}$ in the Sun. This leads to a large range of scales from giant cells on the order of the thickness of the convective layer (e.g. $200 \mathrm{Mm}$ in the Sun) down to granulation patterns $(1 \mathrm{Mm})$, with intermediate sizes such as supergranulation $(20-50 \mathrm{Mm})$ well delineated by the surface magnetic network (Rieutord \& Rincon 2010). As the luminosity (energy output) of the star changes 

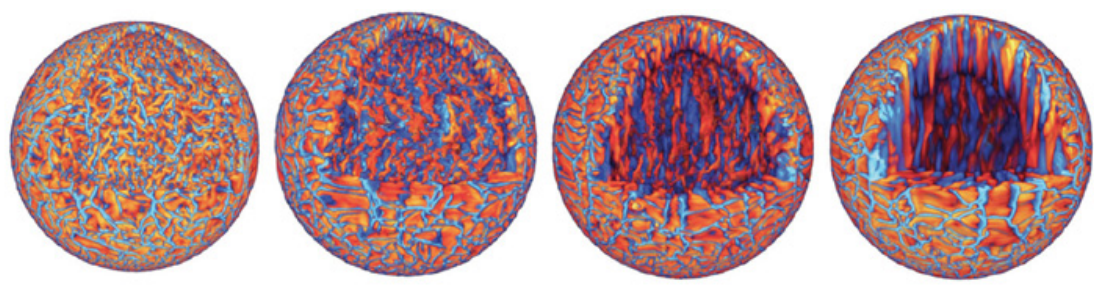

Fig. 5. 3-D rendering of turbulent convection in solar-like stars with various surface envelope thickness spanning radial density contrasts $\Delta_{r} \bar{\rho}_{c z}$ from 13 to 272 (Bessolaz \& Brun 2011). The radial component of the velocity is shown with warm upflows in red tones.

so does the amplitude of the convective flow (cf. Fig. 4). A simple scaling argument based on mixing length theory (MLT) gives $v_{r} \propto\left(L_{*} /\left(\rho_{c z} R_{*}^{2}\right)\right)^{1 / 3}$, with $L_{*}$ and $R_{*}$ respectively the star's luminosity and radius and $\rho_{c z}$ the averaged density of the convective envelope (Kippenhahn et al. 2013; Brun et al. 2015b, 2017). The luminosity varies by at least 2 orders of magnitude across the spectral type we are considering (i.e. from early $\mathrm{F}$ to early $\mathrm{M}$ ) because $L_{*} \propto M_{*}^{4}$ on the main sequence, whereas the stellar radius on the contrary varies much less with stellar mass $\left(R_{*} \propto M_{*}^{0.9}\right)$. It is thus expected that the vigor of the convective flows varies significantly modulo the effect of density. As can been seen in Figure 4, 1-D stellar structure models of solar-like stars indicate that the mass contained in the convective enveloppe, and as a consequence the mean density, changes significantly from $\mathrm{M}$ to $\mathrm{F}$ stellar type stars, in such a way that more massive $\mathrm{F}$ stars have on average a lower mean density than low mass K/M ones (Matt et al. 2011; Brun et al. 2017). This is easily understood as the base of the envelope moves up towards the surface with the thinning of the convective envelope ( $c f$. right panel of Fig. 4). This yields a large amplitude difference between $\mathrm{F}$ and $\mathrm{K} / \mathrm{M}$ stars for their typical convective velocity and the radial extent of their convective cells. Such velocity intensity difference is recovered in global numerical simulations of stellar convection. We illustrate an example of such 3-D turbulent convective simulations in Figure 5, where we clearly see the complex network of downflows surrounding broad upflows and the small convection patterns paving the surface, and how the aspect ratio of the convective enveloppe modifies the radial structure of the flows (Bessolaz \& Brun 2011).

Since all solar-like stars rotate, the turbulent motions within their convective envelopes experience the effect of the Coriolis force to a degree that depends on their fluid Rossby number $R_{o f}=\omega / 2 \Omega_{*}$, here defined as the ratio between turbulent and the planetary vorticities (Pedlosky 1982). We note that several definitions of the Rossby number exist in the community (stellar Rossby based on the ratio between convective turnover time and stellar rotation period; convective Rossby $\sqrt{R a / T a P r}$; local Rossby number), but it can be shown that they depend almost linearly on one another (see for example Kim \& Demarque 1996; Landin et al. 2010; Brun et al. 2017, for a detailed discussion). We here choose to use the fluid Rossby $R_{o f}$ because a clear transition of behavior occurs at $R_{o f} \sim 1$ 
with the differential rotation becoming prograde (i.e. in the direction of the bulk rotation) or retrograde (i.e. in the opposite direction). Indeed if the stars rotate fast enough such that the large scale motions (larger than the Rossby radius of deformation) feel as they rise and sink the continuous action of the Coriolis force then the redistribution of angular momentum is such that a non uniform rotation profile is established in the convective envelope.

In Figure 6 we display 3 realizations of the longitudinally averaged differential rotation and meridional circulation computed with the ASH code (Brun et al. 2017). These profiles have been obtained by changing either the stellar mass (i.e. aspect ratio and luminosity) or the reference frame rotation rate. We note several trends: as the rotation rate is increased, the differential rotation becomes more and more aligned with the rotation axis (along $\hat{e}_{z}$ ) and likewise the meridional circulation possesses more and more cells both in latitude and radius. The 1.1 solar mass case rotating at the solar rate possesses an anti-solar differential rotation, with slow equator and fast pole. It is indeed one of the cases published in the study with a Rossby number greater than 1. Similar behavior have been recently reported in the literature (Bessolaz \& Brun 2011; Matt et al. 2011; Guerrero et al. 2013; Gastine et al. 2014; Käpylä et al. 2014). We thus see that the transition between prograde and retrograde differential rotation will not occur at the same rotation rate $(v \sin i)$ for each stellar spectral type. In Brun et al. (2017), we have derived a simple scaling law relating the Rossby number the star's mass and rotation rate, e.g. $R_{o f} \sim c_{1} M_{*}^{1.7} / \Omega_{*}$ that can be recast as a function of effective temperature $T_{\text {eff }}$, e.g. ${ }^{5} \quad R_{o f} \sim c_{2} T_{\text {eff }}^{3.4} / \Omega_{*}$, with $c_{1}$ and $c_{2}$ constants that can be adjusted such that $R_{o f}$ takes a value between 0.6 and 1 in the solar case and/or through numerical simulations as in (Brun et al. 2017; cf. Fig. 22 and Eq. (33)). We also note the strong shear layer at the base of the convective domain (e.g. the tachocline layer discussed in the previous section, see Spiegel \& Zahn 1992) that naturally develops in each modeled stars with a profile closer to the solar case for all the cases with a Rossby number $R_{o f} \sim 0.6$.

These profiles can be understood by considering the transport of angular momentum in a spherical convective shell. When performing this analysis as in Brun \& Toomre (2002), Brown et al. (2008), Augustson et al. (2012), Featherstone \& Miesch (2015), Brun et al. (2017), one finds that the Reynolds stresses are at the origin of the prograde equator for models with a low Rossby number. Through gyroscopic pumping the meridional circulation transports angular momentum to counterbalance the torque applied by the Reynolds stresses (from which we have subtracted the contribution from viscous effect). We refer to Spiegel \& Zahn (1992), Mathis \& Zahn (2004), McIntyre (2007), Garaud \& Bodenheimer (2010), Brun et al. (2011), Miesch \& Hindman (2011), Featherstone \& Miesch (2015), Passos et al. (2017) for a more thorough discussion of the role of the meridional circulation

5 The derivation is as follows: starting from the stellar luminosity $L_{*}=4 \pi \sigma R_{*}^{2} T_{e f f}^{4}$, assuming $L_{*} \sim M_{*}^{m}$ and $R * \sim M_{*}^{n}$ (with $m=4.6$ and $n=1.3$ from Brun et al. 2017), then $T_{\text {eff }} \sim$ $M_{*}^{(m-2 n) / 4} \sim M_{*}^{0.5}$. All quantities are normalized to solar values, i.e. $M_{*}$ is actually $M_{*} / M_{\odot}$. 

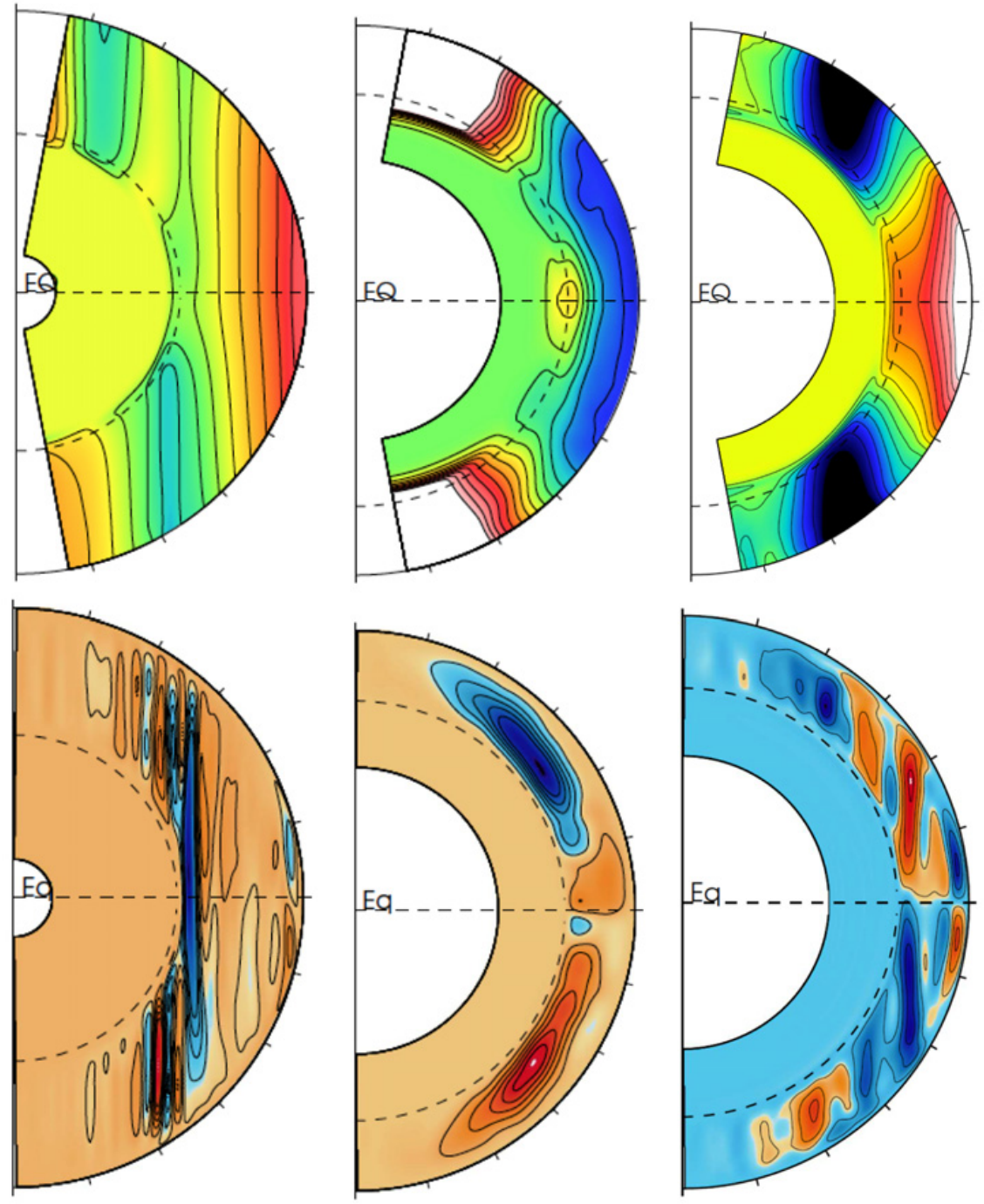

Fig. 6. Differential rotation and meridional circulation realized in 3-D models of solarlike convective envelope coupled nonlinearly to a stably stratified interior (i.e. both zones back react on one another Brun et al. 2017). We show a low mass star model $\left(0.5 M_{\odot}\right)$ rotating at 1 time the solar rate $\Omega_{\odot}$ and two realizations of a high mass star model $\left(1.1 M_{\odot}\right)$ rotating at the solar rate and 3 times faster. Top row display color contours of $\Omega(r, \theta)$ and bottom row the meridional streamfunction. Prograde rotation is shown in red/white colors and retrograde rotation in dark/blue. Clockwise meridional flows in red. 

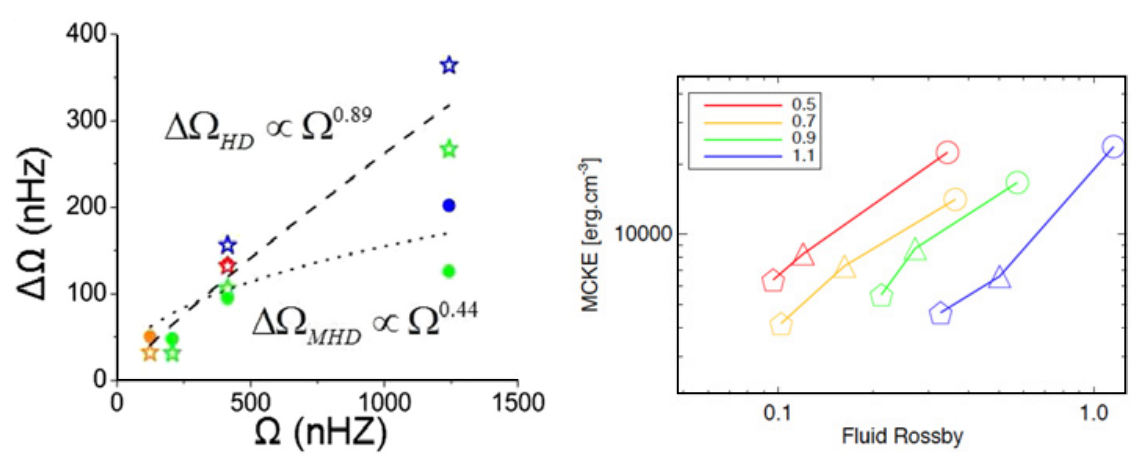

Fig. 7. Left: Differential rotation $\Delta \Omega$ as a function $\Omega_{*}$ for stellar masses ranging from 0.5 up to $1.1 M_{\odot}$ (Varela et al. 2016; Brun et al. 2015b). Shown are the hydro and MHD cases (see next section for the discussion of the later). We note that the MHD cases have a weaker dependence on rotation rate. Right: Kinetic energy of the axisymmetric meridional circulation as a function of the Rossby number for models ranging from 0.5 to $1.1 M_{\odot}$, from Brun et al. (2017). Contrary to what is often assumed in simpler, non self-consistent models, meridional flows tend to be weaker for faster rotation rate.

in reaching angular momentum balance in rotating stars. The orientation of the iso-contours of $\Omega$ from being cylindrical to being more conical (solar-like) at mid latitude is due to the effect of an efficient thermal wind associated with latitudinal entropy variations (with relatively hotter poles and cooler equator) in prograde cases (Miesch et al. 2006). These variations are enhanced further by the presence of a tachocline (Brun et al. 2011). The temperature fluctuations have the opposite latitudinal variations for slowly, retrograde cases, with warm equator and cool poles, and the temperature contrast tends to strongly increase with stellar mass (Brun et al. 2017).

One important aspect of performing 3-D numerical simulations is that one can obtain general trends on the variations of the main physical processes acting in stellar convective envelopes with various parameters such as rotation rate, luminosity, aspect ratio. We focus on scaling laws for the differential rotation and meridional circulation by synthesizing the recently published 3-D stellar convection models computed with the ASH code. The models range from 0.5 up to 1.3 times the solar mass and from 1 to 20 times the solar rotation rate (Brown et al. 2008; Matt et al. 2011; Augustson et al. 2012). In Figure 7 we show how the latitudinal differential rotation contrast $(\Delta \Omega)$ varies as a function of rotation rate (e.g. $\Delta \Omega \propto \Omega_{*}^{n}$ ) for both hydrodynamical cases and MHD cases (see next section). There is a clear trend for $\Delta \Omega$ to become larger with increasing rotation rate. For the hydrodynamical cases this is in qualitative agreement with direct and indirect observational data that imply larger absolute shear rates in rapid rotators to produce stronger magnetic fields and enhanced angular momentum loss relative to slow rotators. The actual slope seems a bit larger than observed by 
(Barnes et al. 2005; Collier Cameron 2007; exponent $n_{*}$ around 0.15 but not so when compared with the work by (Donahue et al. 1996; exponent $n_{*}$ of 0.7). Saar (2011) found that the presence of binaries in the published samples, which can be distinct from single stars because of tidal effects have led to an underestimate of the dependency of $\Delta \Omega$ on rotation rate. More recent studies seem to find a value for the exponent $n_{*}$ near 0.3 (Reinhold \& Gizon 2015) and others even suggest that it is spectral type dependent (Balona \& Abedigamba 2016). Clearly more observational data are required to better constrain the exponent $n_{*}$. We also clearly see that more massive stars possess larger $\Delta \Omega$ for a given Rossby number. This is in reasonable agreement with the observations published in Barnes et al. (2005). Although, given the paucity of data and the rather large error bars, the exact exponent is uncertain. Data from the Kepler mission and in the (future) PLATO mission are/will be a major asset in this context (García et al. 2014; Reinhold \& Gizon 2015; Rauer et al. 2014).

Another interesting property found with 3-D simulations of stellar rotating convection is that the meridional circulation is found to decrease in strength as the stars rotate faster. Rapid rotation causes more kinetic energy to channel into toroidal motions, which can be anticipated by looking at the equation for gyroscopic pumping (Landin et al. 2010; Augustson et al. 2012). This has a very important consequence: flux-transport mean field dynamo models (Charbonneau 2013) can't be easily extended to other stellar spectral type to explain the dependence of their cycle period with their rotation period. Indeed flux-transport models of the advective type depends directly on the meridional circulation amplitude to set the cycle period. Up to very recently, observations were indicating that the cycle period should decrease with increasing rotation rate. However, given that the meridional flow is found to weaken, this can only result in an increase of the activity cycle as was shown by Jouve et al. (2010). One needs either to take into account multi-cellular flows as observed also in 3-D simulations (Jouve et al. 2010) or take into account turbulent pumping (Do Cao \& Brun 2011) to compensate for the slower meridional flow. More recent and better analyzed observations of stellar cycles question the previously established relation between cycle period and rotation period, see Strugarek et al. (2017) and discussion in the next subsection.

Since the coupling between the convective envelop and the radiative interior of solar-like stars is important to model and understand, attempts to model gravity waves excitation and propagation in simulations solving simultaneously the convection and the radiative interior in $3-\mathrm{D}$ have been successfully conducted Alvan et al. (2014b, 2015b). In this recent work, numerical regularization of the solution at $r=0$ has allowed to compute full sphere models. As can be seen in Figure 8, a striking difference exists between the two zones. Along with the usual tilted convective cells surrounded by cold downflow lanes of turbulent stellar convection simulations one can clearly see concentric rings in the deep stably radiative interior (center of the sphere in that solar-like star case). These are gravity waves excited mostly by the pummeling of turbulent plumes at the top of the radiative interior. A detailed study revealed that the waves behave indeed like gravito-inertial waves 

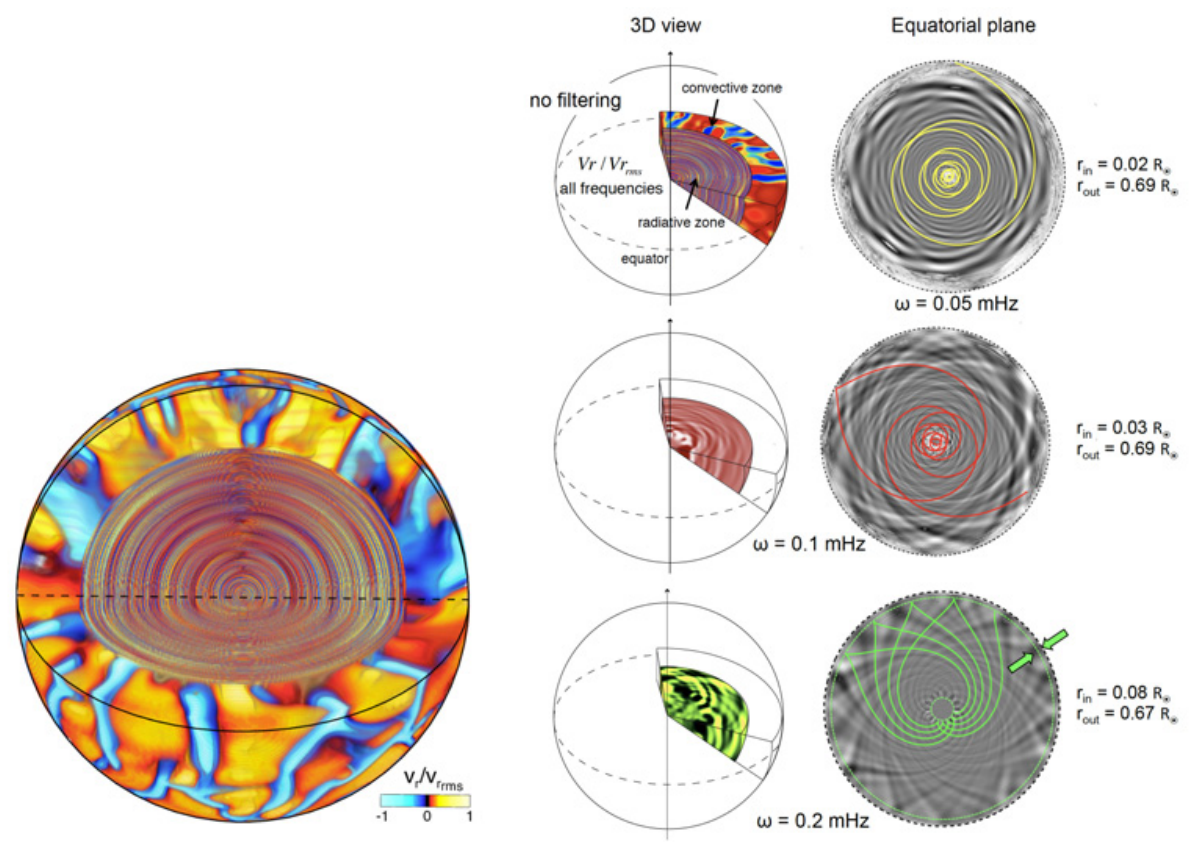

Fig. 8. 3D volume rendering: Gravity waves excited by turbulent convective plumes as seen in a 3-D hydrodynamical simulation of a solar-like star as a whole (from $r=0$ to $0.97 \mathrm{R}$, with the base of the convective envelope located at $r=0.7 \mathrm{R}$; Alvan et al. (2014b, 2015b). Red colors correspond to upflows or outward propagating waves. Right: Temporal filtering for various frequencies showing that all type of gravity modes (low or high frequency) co-exist within the radiative interior. Superimposed to the gray shade equatorial slices coming from the numerical simulations are ray path drawings at the appropriate frequency. For high frequency we recover the cusp-like structure of the wave path.

(in this purely hydrodynamical setup). When the simulation is filtered in time and a frequency close to the maximal Brunt-Vasaila frequency of the model is selected, one recovers the more classical cusp-like shape of the waves (ray path) ( $c f$. Fig. 8 right panel). Gravity waves were a topic of key interest for Jean-Paul as discussed in Zahn et al. (1997), Kumar et al. (1999).

\subsection{Stellar dynamos and magnetism}

Stars like the Sun exhibit a large range of magnetic phenomena, starspots and flares being the more obvious. Further, the observed magnetic activity seems to possess in some instance a cyclic behavior akin to the 11-yr cycle of the Sun. Period of cycles seem to depend on stellar spectral type and rotation rate. An obvious trend is that old (older than the age of the Hyades cluster $\sim 800 \mathrm{Myr}$ ), slowly 


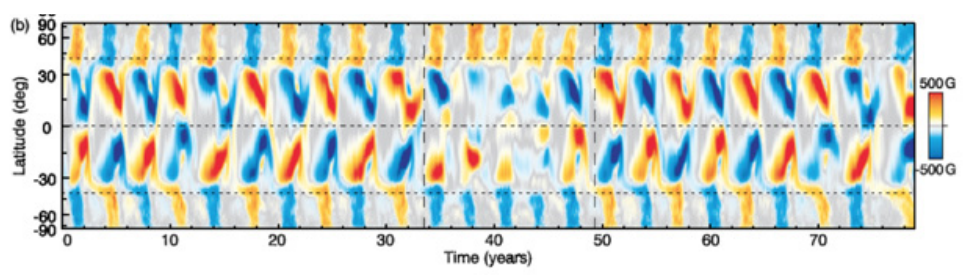

Fig. 9. Butterfly diagram (time-latitude contour plot showing the toroidal magnetic field at the base of the convective enveloppe) in the K3s simulation of Augustson et al. (2015). Clear dipolar (antisymmetric with respect to the equator) dominated cyclic magnetic activity is visible except for a period of 20 year when the simulation undergoes a sudden change of dominant mode and a reduction of field amplitude akin to grand minima such as solar Maunder minimum.

rotating solar-like stars are less active than their younger counterparts. We have also discussed in the previous section that convection and large scale flows change with spectral type. It is certainly not surprising that the associated dynamo action and magnetic activity do so as well.

In solar-like stars, along with the degree of turbulence, rotation plays an important role in determining the global properties of their magnetism. This is due to a shift in the balance of forces driving the flow between the advection, Coriolis and Lorentz terms. As the rotation rate increases the Lorentz force tends to balance the Coriolis force yielding larger magnetic energy in superequipartion with the kinetic energy of the flow (a direct consequence of a magnetostrophic state) as in the Earth's iron core. The Elsasser number $\Lambda=B^{2} / 4 \pi \bar{\rho}_{c z} \eta \Omega_{0}$, with $\bar{\rho}_{c z}$ the mean density in the convective envelope, $\eta$ the magnetic diffusivity, $\Omega_{0}$ the stellar rotation rate, $B$ a characteristic magnetic field of the $\mathrm{CZ}$, is useful to discuss this balance of terms in the Navier-Stokes (N.V.) equation. Depending on the amplitude of this number and on the balance assumed in the Navier-Stokes equation, various scalings of the magnetic field amplitude can be expected (Fauve \& Pétrélis 2007; Christensen 2010; Brun et al. 2015a).

Several groups have recently published extensive parameter studies of dynamo action in rotating convective spherical shells of various thickness and degree of stratification. The key results can be summarized as follow: 3-D numerical simulations of dynamo action in solar-like stars have revealed a large range of behavior, from steady dynamo, to irregular and cyclic ones and references therein (Brun et al. 2004; Brown et al. 2010, 2011; Racine et al. 2011; Gastine et al. 2012; Schrinner et al. 2012; Augustson et al. 2013; Käpylä et al. 2013; Nelson et al. 2013; Schrinner et al. 2014; Augustson et al. 2015; Guerrero et al. 2016; Strugarek et al. 2017; Brun \& Browning 2017). In Figure 9 we display a latitude-time diagram of the toroidal magnetic field in one of the recent 3-D nonlinear cyclic dynamo solutions published in Augustson et al. (2015).

For low mass (M-type) stars, it is seen that a bistability similar to that found for the geodynamo is possible. Observations by Morin et al. (2010) seems to 
indicate that a strong dipolar state and a multipolar state may coexist for stars having similar stellar parameters. Dynamo models show that around a local Rossby number of 0.1 a weak (multipolar) and strong (dipolar) dynamo branch may coexist (Christensen \& Aubert 2006; Morin et al. 2011). Such bistable states are found in a given range of parameters (low stratification, high $\mathrm{Pm}$ ) and may not actually exist in real stars. It may be the case that these stars are observed in a different phase (low vs. high) of their activity cycle. Other similarity between the geodynamo and low mass stellar dynamos also exist such as the scaling of the magnetic energy with the available heat flux (Christensen et al. 2009). Such scaling tends to break down for higher mass stars for which the tachocline may play a significant role.

Others 3-D simulations of $\mathrm{F}, \mathrm{G}$ and $\mathrm{K}$ stars show that for high rotation rates, large scale magnetic wreaths (see Fig. 10 left panel) are obtained without requiring the presence of a tachocline (Brown et al. 2010, 2011; Nelson et al. 2013). It is found that as the model is made more turbulent, the steady magnetic wreaths become more time dependent and can lead to cyclic activity and even to $\Omega$-loop that are thought to be at the source of flux emergence and starspots. The existence of starspots in 3-D global dynamo simulations is the most challenging aspect of recent developments, and has been called the spot-dynamo paradox (Brun \& Browning 2017). Currently no global-scale 3-D nonlinear MHD dynamos develop spots. Global flux emergence simulations have been computed to assess the role of the Coriolis force, Hoop stresses, convective motions, large scale mean flows and background magnetic fields on the evolution and morphology of simplified active regions by varying the properties of idealized global flux ropes (Fan 2009; Jouve \& Brun 2009; Weber et al. 2011; Iş1k et al. 2011; Jouve et al. 2013; Pinto \& Brun 2013; Weber et al. 2013) but until very recently, none had self-consistently dealt with the formation of sunspots in a cyclic convective dynamo. So in order to be able to physically characterize the saturation of $B$ and $f$ independently, spotdynamos - wherein dynamos self consistently produce rising omega-loops - must be developed and the parameter space explored systematically.

There has been recent progress in this direction using improved numerical treatment of the diffusivities in 3-D simulations (via dynamical Smagorinsky, slope limited diffusion or implicit large eddy simulation-ILES). Authors of these studies have been able to lower the diffusivity level low enough to yield more time dependent dynamo solutions possessing both cyclic behavior, regular butterfly diagram and torsional oscillation-like behavior (Käpylä et al. 2011, 2012, 2013; Dubé \& Charbonneau 2013; Smolarkiewicz \& Charbonneau 2013; Beaudoin et al. 2013; Augustson et al. 2013). Some have even obtained for the first time buoyant magnetic wreaths (Nelson et al. 2011, 2013). In these recent simulations, magnetic wreath-like structures become turbulent and intermittent enough, that many intense bundles of fields reaching $50 \mathrm{kG}$ start to form $\Omega$-loop like structures with statistical properties for rising loops that qualitatively agree with observations (Nelson et al. 2014). We believe that such simulations are the progenitors of future more realistic spot-dynamos (for a first step in this direction, see the simulations of flux emergence from a dynamo simulation by Chen et al. 2017) that 


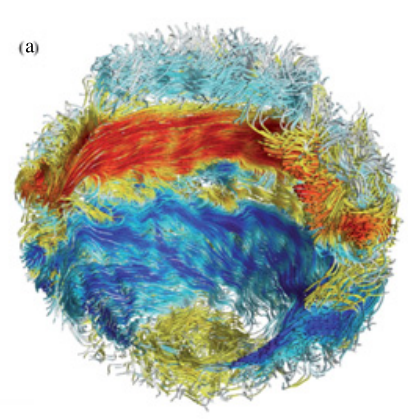

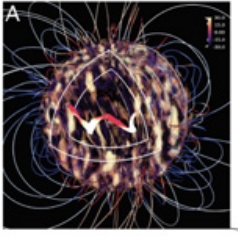
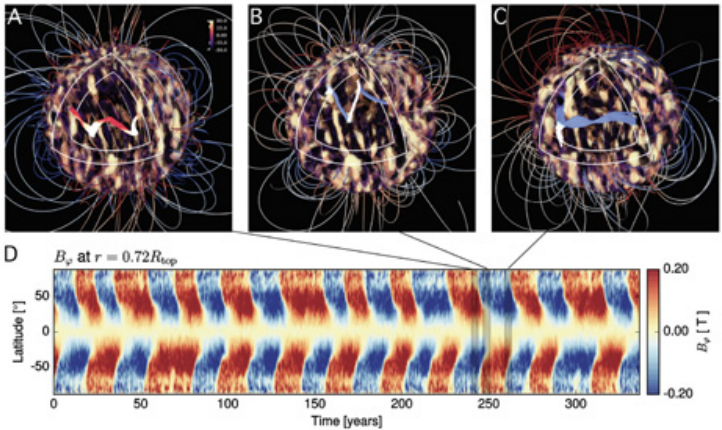

Fig. 10. Left: Magnetic wreaths shown by 3-D rendering of magnetic field lines (Brown et al. 2010). Right: Temporal sequence of $3-\mathrm{D}$ views showing the magnetic field realized in series of solar-like star simulation published in Science (Strugarek et al. 2017) which was further extrapolated using potentiel field approximation to about 1.5 stellar radius. Note the global polarity field reversal in both field components.

will allow us to characterize the link between dynamo action, flux emergence and large scale magnetic topology as a function of stellar parameters.

In recent numerical 3-D MHD simulation studies computed with ASH and EULAG-MHD (Augustson et al. 2015; Strugarek et al. 2017), the authors have shown that non linear feedback can induce very interesting behavior in convective dynamos. In the first study, grand minima states have been identified ( $c f$. Fig. 9, around year 40), the cyclic convective dynamo going out and back on its regular dipolar dominated behavior. The interplay between antisymmetric and symmetric dynamo families and a low magnetic Prandtl number seems to be playing a key role in this intermittent behavior (see also discussion in DeRosa et al. 2012 and references therein). In the second study, the interplay of the large scale differential rotation and the magnetic field is what actually causes the reversal, with a local change of sign of the latitudinal shear that induces an opposite sign toroidal field and hence a reversal of the dynamo cycle ( $c f$. Fig. 10 right panel). Further, for the first time a systematic variation of the period of the stellar magnetic cycle has been observed, pointing to a possible new scaling of the cycle period with the rotation period, opposite to that provided by kinematic $\alpha-\omega$ dynamo, e.g. longer cycle period for faster rotation.

Differential rotation is modified by the presence of a dynamo field. The influence of the Lorentz force on the convective flow exerts a torque that tends to rigidify the convective envelope. Often it is found that it is near the cold vortex (cyclonic plumes) that the Maxwell stresses are the more intense, as the field is being swept towards the down flow lanes. Large scale magnetic torques can also come into play (as a "magnetic net" that slows down the large scale azimuthal flow), this depends on whether or not the simulations have developed an intense large scale dipolar component (Brun 2004; Schrinner et al. 2012). In Figure 11 we 

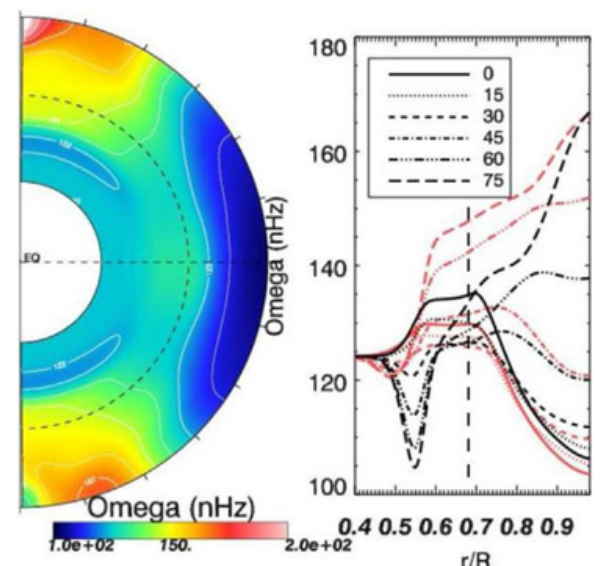

$\mathrm{r} / \mathrm{R}$

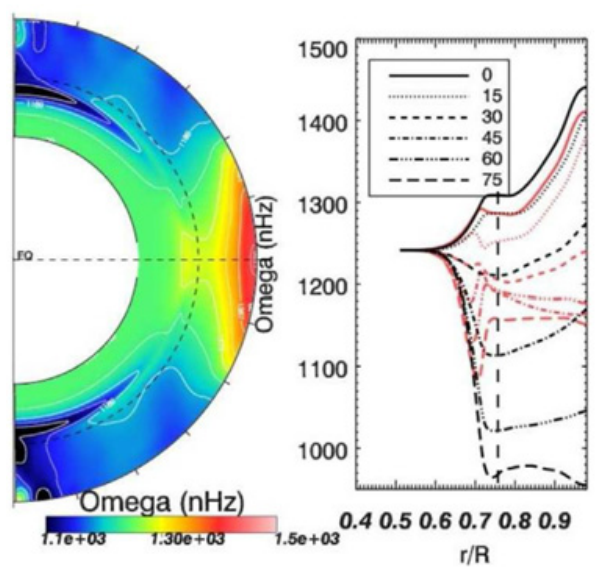

$\mathrm{r} / \mathrm{R}$

Fig. 11. Differential rotation in 3-D dynamo models of solar-like convective envelope coupled nonlinearly to a stably stratified interior (Varela et al. 2016; Brun et al. 2017). We show a retrograde low mass star model $\left(0.7 M_{\odot}\right)$ rotating at $1 / 4$ time the solar rate $\Omega_{\odot}$ and one high mass prograde star model $\left(1.1 M_{\odot}\right)$ rotating at 3 times the solar rate. Left part on each panel display color contours of $\Omega(r, \theta)$ and right part the cut at indicated latitude, comparing the MHD dynamo case (red curves) to its purely hydrodynamic progenitor (black curves). Prograde rotation is shown in red/white colors and retrograde rotation in dark/blue.

display how the differential rotation is modified in two dynamo simulations that are the generalisation of the cases discussed in the previous section in the hydrodynamical mode and in more details in (Brun et al. 2017). Since it is unlikely that solar-like stars do not possess intense magnetic fields, the state of differential rotation of these stars is certainly described with better fidelity by the MHD cases shown in Figure 11 and discussed in Varela et al. (2016). Of particular interest is the trend that these nonlinear convection models predict for how the surface differential rotation contrast varies with rotation rate or stellar mass. In the left panel of Figure 7 we also display how the presence of magnetic field modifies the scaling relation between $\Delta \Omega$ and $\Omega$. We find that overall the MHD models are in better agreement with observations. Indeed the trend (or exponent $n_{*}$ ) vs. rotation rate is weaker.

Finally we also note that the torque applied by the stellar wind depends mostly on magnetic fields whose geometry is expected to change as a function of stellar cycle and overall rotation rate (see Pinto et al. 2011; Réville et al. 2014; Réville et al. 2016). Hence assessing the large-scale topology of stellar magnetic fields and how it changes as a function of stellar parameters is very important as it has a direct bearing on stellar evolution. 


\section{Perspectives}

The characterization of the many aspects of stellar dynamics (convection, rotation, magnetism) has made significant progress over the last two decades. We have moved away from a quasi-static view of stars to a time dependent, turbulent and magnetic description of these fascinating objects. Asteroseismology has significantly contributed to this progress, along with spectropolarimetry and ever improving numerical simulations and models of stellar convection and dynamo action. However much remains to be done. Most of the hypothesis have been deduced by extrapolating our (partial) understanding of the Sun, to other solarlike stars. The continuous back and forth comparison between the Sun and other stars has been most productive and will continue being so with many projects relying both on ground-based telescopes and space-born missions in the planning. The launch of ESA's M3 Cosmic Vision PLATO in 2026 (Rauer et al. 2014) will continue the data collection initiated by its predecessors Corot, Kepler, TESS, helping to improve our knowledge of stellar dynamics and magnetism. Likewise spectro-polarimeters such as SPIROU@CFHT will provide high quality observations of stellar magnetism and the interaction of this intense stellar activity with close-in planets.

On the theoretical side, the recent progress on analytical work and multi-D models of stars of various mass, rotation rates and age, has allowed for a direct comparison with spectropolarimetric and asteroseismic data. Simulations coupling the angular evolution and magnetic activity on long time scale are in the making and should help explain the rotational history of stars. Acoustic and gravity modes excitation and propagation are now computed in detail and stellar cycles have now been found in many self-consistent convective dynamo simulations. General trends such as getting higher activity levels with rotation rate have been recovered. But questions about the relation between cycle period and rotation period have been put on the table, as nonlinear simulations seem to have a different dependency than classical $\alpha-\omega$ dynamo. Still the exact dynamo mechanisms at work and resulting magnetic properties (geometry, intensity, variability, cycle period) for any given star still need intense work. This comes about because the relative importance, spatial and temporal phasing and nonlinear interactions of the identified processes (e.g. shear in the tachocline, $\alpha$ and $\omega$ effects, stability of the magnetic wreaths, role of the meridional flow, energy channeling between various reservoir (internal, potential, kinetic, magnetic) and turbulent pumping) at the origin of the organisation of the global (large scale) dynamo and the establishment of a cyclic magnetic activity vary. Thus the characterization of the exact dependence of the stellar dynamo properties to stellar parameters such as mass, luminosity, rotation/age and stratification is still an evolving understanding (Strugarek et al. 2017). In order to progress further theoretical models and high performance multi-D numerical simulations will benefit from having the following inputs/constraints:

- better constrained variations of the differential rotation in solar-like stars as a function of stellar parameters (mass, rotation/age, metallicity) and its latitudinal and radial profiles 
- improved characterization of the $P_{c y c}$ vs. $P_{\text {rot }}$ relation, both on the observational and theoretical sides

- some assessment of the surface horizontal flows

- convective power and extent of the convective envelopes

- improved probing of the solar tachocline and its time dependancy with helioseismology

- link between star spot numbers and stellar parameters, relative contribution to stellar brightness between dark spot, and bright faculae

- high cadence spectropolarimetric maps over the course of many years hence possibly mapping stellar cycles for many different stars

- calibration with asteroseismology of gyro-chronology by studying stellar clusters of various ages

- coupling between inner dynamics and surface dynamics

- development of new numerical 3-D stellar MHD codes ready for Exa-scale class super computers.

We are hopeful that in the next decade or so, these multitude of information will become available and will aid the community in constraining and further developing modern theories and computational models of solar and stellar activity. When this will occur they will be a great tribute to Jean-Paul's vision that stars are dynamical magnetized objects that need to be fully and nonlinearly treated as such!

\section{Tribute to Jean-Paul written on the 31st of July 2015 for the Père Lachaise ceremony}

Jean-Paul, mon Jean-Paul, que c'est dur d'écrire ces mots, que cette nouvelle effroyable survenue un matin à $6 \mathrm{~h}$ à Boulder le 15 juillet 2015 dans la maison de Katherine tout en haut des montagnes par un coup de fil de Juri, ton ami de plus de 40 ans, a été dévastatrice, un choc frontal, un big crunch cosmique, dont les sursauts se font encore sentir à l'infini et surtout au quotidien, laissant un vide indicible ... Jean-Paul que la maladie est cruelle emportant toujours les meilleurs d'entre nous, Jean-Paul apprendre cette nouvelle à Boulder, où tout me rappelle ta présence et nos nombreuses discussions quand tu venais à JILA durant ma période de postdoc là bas, est tellement douloureux, Jean-Paul, cette impuissance de ne pouvoir être là aujourd'hui (car aux USA en partance pour l'Assemblée Générale à Hawaii) pour te rendre hommage rajoute à ma peine immense, à ma tristesse incommensurable... Je remercie Agnès mon épouse, que toi et Suzy avez toujours accueilli à bras ouverts ainsi que mes enfants, de venir me représenter en ce jour si difficile pour nous tous. Suzy, sache que je (nous) partage(ons) ton immense 
peine et que tu peux compter sur nous dans cette épreuve si dure, Jean-Paul, mon guide, mon mentor, mon papa scientifique, tu m'as tellement appris, transformant le fougeux jeune homme, en comment dire ... un scientifique plus posé et réfléchi ... Je me rappelle les répétitions d'oraux des concours à Santa Barbara en 2000, que de chemin parcouru ensemble depuis, Jean-Paul, le "Convective Collective" vient de perdre un de ses membres éminents, je me rappelle ces 2 mois au Newton Institute en 2004 avec Ed, Juri, Douglas, jonglant avec les équations, les concepts de dynamiques astrophysiques les plus subtils, que ces joutes intellectuelles vont me manquer ! Jean-Paul, le laboratoire que nous avons constitué avec Stéphane au Service d'Astrophysique du CEA-Saclay, on te le doit, tu nous as tout appris, c'est le tien, tu en es et tu en resteras son icône parternelle et scientifique, montrant la voie tel un phare dans la nuit cosmique, et j'espère de tout coeur que nous continuerons à faire honneur à ton école de pensée en dynamique des fluides astrophysiques, nos succés ont été, sont et seront les tiens à tout jamais. Jean-Paul, je me rappelle les nombreux repas chez toi ou à la Rotonde où tu venais partager un bon repas et du bon vin rencontrer nos jeunes, et nous donner tes conseils avisés pour notre recherche à tous. Jean-Paul, ta gentillesse, ta bienveillance, ton éthique, ta patience, ton écoute, ta modestie, ton humilité sont tant d'exemples à suivre.

Jean-Paul, mon ami, tu me manques, tu me manqueras, je ne t'oublierai jamais. Sacha, Boulder le 31 Juillet 2015.

\section{References}

Acevedo-Arreguin, L.A., Garaud, P., \& Wood, T.S., 2013, MNRAS, 434(1), 720

Alvan, L., Brun, A.S., \& Mathis, S., 2014a, Astron. Astrophys., 565, A42

Alvan, L., Brun, A.S., \& Mathis, S., 2014b, Astron. Astrophys., 565, A42

Alvan, L., Strugarek, A., Brun, A.S., Mathis, S., \& Garcia, R.A., 2015a, Astron. Astrophys., 581, A112

Alvan, L., Strugarek, A., Brun, A.S., Mathis, S., \& Garcia, R.A., 2015b, Astron. Astrophys., 581, A112

Augustson, K., Brun, A.S., Miesch, M., \& Toomre, J., 2015, Astrophys. J., 809, 149

Augustson, K.C., Brown, B.P., Brun, A.S., Miesch, M.S., \& Toomre, J., 2012, Astrophys. J., $756(2), 169$

Augustson, K.C., Brun, A.S., \& Toomre, J., 2013, Astrophys. J., 777, 153

Aurière, M., Wade, G.A., Silvester, J., et al., 2007, Astron. Astrophys., 475(3), 1053

Baliunas, S.L., Donahue, R.A., Soon, W.H., et al., 1995, Astrophys. J., 438, 269

Ballot, J., Brun, A.S., \& Turck-Chièze, S., 2007, Astrophys. J., 669, 1190

Balona, L.A., \& Abedigamba, O.P., 2016, MNRAS, 461, 497

Barnabé, R., Strugarek, A., Charbonneau, P., Brun, A.S., \& Zahn, J.-P., 2017, Astron. Astrophys., 601, A47

Barnes, J.R., Collier Cameron, A., Donati, J.-F., et al., 2005, MNRAS, 357, L1

Basu, S., 1997, MNRAS, 288, 572

Basu, S., \& Antia, H.M., 2003, Astrophys. J., 585, 553 
Beaudoin, P., Charbonneau, P., Racine, E., \& Smolarkiewicz, P.K., 2013, Sol. Phys., 282, 335

Bessolaz, N., \& Brun, A.S., 2011, Astrophys. J., 728, 115

Braithwaite, J., \& Spruit, H.C., 2017, Open Sci., 4, 160271

Brandenburg, A., \& Dobler, W., 2002, Comput. Phys. Commun., 147, 471

Brown, B.P., Browning, M.K., Brun, A.S., Miesch, M.S., \& Toomre, J., 2008, Astrophys. J., 689, 1354

Brown, B.P., Browning, M.K., Brun, A.S., Miesch, M.S., \& Toomre, J., 2010, Astrophys. J., 711,424

Brown, B.P., Miesch, M.S., Browning, M.K., Brun, A.S., \& Toomre, J., 2011, Astrophys. J., 731,69

Brown, T.M., Christensen-Dalsgaard, J., Dziembowski, W.A., et al., 1989, Astrophys. J., 343,526

Browning, M.K., 2008, Astrophys. J., 676, 1262

Browning, M.K., Brun, A.S., \& Toomre, J., 2004, Astrophys. J., 601, 512

Browning, M.K., Miesch, M.S., Brun, A.S., \& Toomre, J., 2006, Astrophys. J., 648, L157

Browning, M.K., Miesch, M.S., Brun, A.S., \& Toomre, J., 2006, Astrophys. J. Lett., 648, L157

Brun, A.S., 2004, Sol. Phys., 220, 333

Brun, A.S., \& Browning, M.K., 2017, Living Rev. Sol. Phys., 14, 4

Brun, A.S., Browning, M.K., Dikpati, M., Hotta, H., \& Strugarek, A., 2015a, Space Sci. Rev., 196, 101

Brun, A.S., Garcia, R.A., Houdek, G., Nandy, D., \& Pinsonneault, M., 2015b, Space Sci. Rev., 196, 303

Brun, A.S., Miesch, M.S., \& Toomre, J., 2004, Astrophys. J., 614, 1073

Brun, A.S., Miesch, M.S., \& Toomre, J., 2011, Astrophys. J., 742, 79

Brun, A.S., Strugarek, A., Varela, J., et al., 2017, Astrophys. J., 836, 192

Brun, A.S., Strugarek, A., Varela, J., et al., 2017, Astrophys. J., 836, 192

Brun, A.S., \& Toomre, J., 2002, Astrophys. J., 570, 865

Brun, A.S., \& Zahn, J.-P., 2006, Astron. Astrophys., 457, 665

Cally, P.S., Dikpati, M., \& Gilman, P.A., 2003, Astrophys. J., 582, 1190

Cattaneo, F., 1999, Astrophys. J., 515, L39

Charbonneau, P., 2013, Saas-Fee Advanced Courses, Vol. 39, Solar and Stellar Dynamos, ed. O. Steiner (Springer, Berlin, Heidelberg)

Charbonneau, P., 2013, Soc. Astron. Sci. Annu. Symp., 39, 187

Charbonneau, P., Christensen-Dalsgaard, J., Henning, R., et al., 1999a, Astrophys. J., 527,445

Charbonneau, P., Dikpati, M., \& Gilman, P.A., 1999b, Astrophys. J., 526, 523

Chen, F., Rempel, M., \& Fan, Y., 2017, Astrophys. J., 846, 149

Christensen, U.R., 2010, Space Sci. Rev., 152, 565

Christensen, U.R. \& Aubert, J., 2006, Geophys. J. Int., 166, 97

Christensen, U.R., Holzwarth, V., \& Reiners, A., 2009, Nature, 457, 167

Collier Cameron, A., 2007, Astronomische Nachrichten, 328, 1030 
Corbard, T., Blanc-Féraud, L., Berthomieu, G., \& Provost, J., 1999, Astron. Astrophys., 344,696

DeRosa, M.L., Brun, A.S., \& Hoeksema, J.T., 2012, Astrophys. J., 757, 96

Dikpati, M., 2012, Astrophys. J. Supp., 745, 128

Dikpati, M., Gilman, P.A., Cally, P.S., \& Miesch, M.S., 2009, Astrophys. J., 692, 1421

Dikpati, M., Gilman, P.A., \& MacGregor, K.B., 2006, Astrophys. J., 638, 564

Do Cao, O., \& Brun, A.S., 2011, Astronomische Nachrichten, 332, 907

Donahue, R.A., Saar, S.H., \& Baliunas, S.L., 1996, Astrophys. J., 466, 384

Donati, J.-F., \& Landstreet, J.D., 2009, Annu. Rev. A\&A, 47, 333

Dubé, C., \& Charbonneau, P., 2013, Astrophys. J., 775, 69

Egeland, R., Soon, W., Baliunas, S., Hall, J.C., \& Henry, G.W., 2017, Living Around Active Stars, 328, 329

Elliott, J.R., \& Gough, D.O., 1999, Astrophys. J., 516, 475

Fan, Y., 2009, Living Rev. Sol. Phys., 6, 4

Fauve, S., \& Pétrélis, F., 2007, Comptes Rendus Physique, 8, 87

Featherstone, N.A., \& Miesch, M.S., 2015, Astrophys. J., 804, 67

Ferraro, V.C.A., 1937, MNRAS, 97, 458

Forgács-Dajka, E., 2004, Astron. Astrophys., 413, 1143

Forgács-Dajka, E., \& Petrovay, K., 2001, Sol. Phys., 203, 195

Forgács-Dajka, E., \& Petrovay, K., 2002, Astron. Astrophys., 389, 629

Fossat, E., Boumier, P., Corbard, T., et al., 2017, Astron. Astrophys., 604, A40

Garaud, P., 1999, MNRAS, 304, 583

Garaud, P., 2002, MNRAS, 329, 1

Garaud, P., \& Acevedo Arreguin, L.A., 2009, Astrophys. J., 704, 1

Garaud, P., \& Bodenheimer, P., 2010, Astrophys. J., 719, 313

Garaud, P., \& Brummell, N.H., 2008, Astrophys. J., 674, 498

Garaud, P., \& Garaud, J.-D., 2008, MNRAS, 391, 1239

García, R.A., Ceillier, T., Salabert, D., et al., 2014, Astron. Astrophys., 572, A34

Garcia, R.A., Mathur, S., Salabert, D., et al., 2010, Science, 329, 1032

Garcia, R.A., Turck-Chièze, S., Jiménez-Reyes, S.J., et al., 2007, Science, 316, 1591

Gastine, T., Duarte, L., \& Wicht, J., 2012, Astron. Astrophys., 546, A19

Gastine, T., Wicht, J., \& Aurnou, J.M., 2013, Icarus, 225, 156

Gastine, T., Yadav, R.K., Morin, J., Reiners, A., \& Wicht, J., 2014, MNRAS, 438, L76

Ghizaru, M., Charbonneau, P., \& Smolarkiewicz, P.K., 2010, Astrophys. J., 715, L133

Gilman, P.A., 1983, Astrophys. J. Supp., 53, 243

Gilman, P.A., 2000, Sol. Phys., 192, 27

Gilman, P.A., 2017, Astrophys. J., 842, 130

Gilman, P.A., Dikpati, M., \& Miesch, M.S., 2007, Astrophys. J. Supp., 170, 203

Gilman, P.A., \& Fox, P.A., 1997, Astrophys. J., 484, 439

Gilman, P.A., \& Miller, J., 1981, Astrophys. J. Supp., 46, 211

Glatzmaier, G.A., 1984, J. Comput. Phys., 55, 461

Glatzmaier, G.A., 1985, Astrophys. J., 291, 300 
Glatzmaier, G.A., 1985, Geophys. Astrophy. Fluid Dyn., 31, 137

Glatzmaier, G.A., \& Gilman, P.A., 1982, Astrophys. J., 256, 316

Gough, D.O., 1969, J. Atmos. Sci., 26, 448

Gough, D.O., \& McIntyre, M.E., 1998, Nature, 394, 755

Guerrero, G., Smolarkiewicz, P.K., de Gouveia Dal Pino, E.M., Kosovichev, A.G., \& Mansour, N.N., 2016, Astrophys. J., 819, 104

Guerrero, G., Smolarkiewicz, P.K., Kosovichev, A.G., \& Mansour, N.N., 2013, Astrophys. J., 779,176

Hall, J.C., Henry, G.W., Lockwood, G.W., Skiff, B.A., \& Saar, S.H., 2009, Astron. J., 138,312

Hotta, H., 2017, Astrophys. J., 843, 52

Hotta, H., Rempel, M., \& Yokoyama, T., 2015, Astrophys. J., 803, 42

Hotta, H., Rempel, M., \& Yokoyama, T., 2016, Science, 351, 1427

Hotta, H., \& Yokoyama, T., 2011, Astrophys. J., 740, 12

Hurlburt, N.E., Toomre, J., Massaguer, J.M., \& Zahn, J.-P., 1994, Astrophys. J., 421, 245

Işık, E., Schmitt, D., \& Schüssler, M., 2011, Astron. Astrophys., 528, A135

Jones, C.A., Boronski, P., Brun, A.S., et al., 2011, Icarus, 216, 120

Jouve, L., Brown, B.P., \& Brun, A.S., 2010, Astron. Astrophys., 509, A32

Jouve, L., \& Brun, A.S., 2009, Astrophys. J., 701, 1300

Jouve, L., Brun, A.S., \& Aulanier, G., 2013, Astrophys. J., 762, 4

Jouve, L., Gastine, T., \& Lignières, F., 2015, Astron. Astrophys., 575, A106

Kageyama, A., \& Sato, T., 1997, Phys. Rev. E., 55, 4617

Kageyama, A., \& Sato, T., 2004, Geochem. Geophys. Geosyst., 5, 9005

Käpylä, M.J., Käpylä, P.J., Olspert, N., et al., 2016, Astron. Astrophys., 589, A56

Käpylä, P.J., Käpylä, M.J., \& Brandenburg, A., 2014, Astron. Astrophys., 570, A43

Käpylä, P.J., Käpylä, M.J., Olspert, N., Warnecke, J., \& Brandenburg, A., 2017, Astron. Astrophys., 599, A4

Käpylä, P.J., Mantere, M.J., \& Brandenburg, A., 2012, Astrophys. J., 755, L22

Käpylä, P.J., Mantere, M.J., Cole, E., Warnecke, J., \& Brandenburg, A., 2013, Astrophys. J., 778,41

Käpylä, P.J., Mantere, M.J., Guerrero, G., Brandenburg, A., \& Chatterjee, P., 2011, Astron. Astrophys., 531, A162

Karak, B.B., Käpylä, P.J., Käpylä, M.J., et al., 2015, Astron. Astrophys., 576, A26

Karak, B.B., \& Petrovay, K., 2013, Sol. Phys., 282, 321

Kim, E.-J., \& Leprovost, N., 2007, Astron. Astrophys., 468, 1025

Kim, Y.-C., \& Demarque, P., 1996, Astrophys. J., 457, 340

Kimura, K., Takehiro, S.-i., \& Yamada, M., 2011, PoF, 23, 074101

Kippenhahn, R., Weigert, A., \& Weiss, A., 2013, Stellar Structure and Evolution

Kitchatinov, L.L., \& Olemskoy, S.V., 2012, MNRAS, 423, 3344

Kosovichev, A.G., 1996, Astrophys. J., 469, L61

Küker, M., \& Rüdiger, G., 2008, J. Phys. Conf. Ser., 118, 012029

Kumar, P., Talon, S., \& Zahn, J.-P., 1999, Astrophys. J., 520, 859 
Landin, N.R., Mendes, L.T.S., \& Vaz, L.P.R., 2010, Astron. Astrophys., 510, A46

Latour, J., Toomre, J., \& Zahn, J.-P., 1981, Astrophys. J., 248, 1081

Lawson, N., Strugarek, A., \& Charbonneau, P., 2015, Astrophys. J., 813, 95

Leprovost, N., \& Kim, E.-J., 2006, Astron. Astrophys., 456, 617

Leprovost, N., \& Kim, E.-J., 2009, Phys. Rev. E., 80, 26302

Masada, Y., Yamada, K., \& Kageyama, A., 2013, Astrophys. J., 778, 11

Mathis, S., \& Zahn, J.-P., 2004, Astron. Astrophys., 425, 229

Matt, S.P., Do Cao, O., Brown, B.P., \& Brun, A.S., 2011, Astronomische Nachrichten, 332,897

McIntyre, M.E., 2007, The Solar Tachocline, 183

Mestel, L., Tayler, R.J., \& Moss, D.L., 1988, Mon. Not. R. Astron. Soc. (ISSN 0035-8711), 231,873

Mestel, L., \& Weiss, N.O., 1987, Mon. Not. R. Astron. Soc. (ISSN 0035-8711), 226, 123

Miesch, M.S., 2001, Astrophys. J., 562, 1058

Miesch, M.S., 2003, Astrophys. J., 586, 663

Miesch, M.S., 2005, Liv. Rev. Sol. Phys., 2, 1

Miesch, M.S., 2007, Astrophys. J., 658, L131

Miesch, M.S., \& Brown, B.P., 2012, Astrophys. J. Lett., 746, L26

Miesch, M.S., Brun, A.S., De Rosa, M.L., \& Toomre, J., 2008, Astrophys. J., 673, 557

Miesch, M.S., Brun, A.S., \& Toomre, J., 2006, Astrophys. J., 641, 618

Miesch, M.S., Elliott, J.R., Toomre, J., et al., 2000, Astrophys. J., 532, 593

Miesch, M.S., \& Hindman, B.W., 2011, Astrophys. J., 743, 79

Miesch, M.S., \& Toomre, J., 2009, Annu. Rev. Fluid Mech., 41, 317

Mitra, D., Tavakol, R., Brandenburg, A., \& Moss, D., 2009, Astrophys. J., 697, 923

Morel, P., 1997, Astron. Astrophys. Supp., 124, 597

Morin, J., Donati, J.-F., Petit, P., et al., 2010, MNRAS, 407, 2269

Morin, J., Dormy, E., Schrinner, M., \& Donati, J.-F., 2011, MNRAS, 418, L133

Nelson, N.J., Brown, B.P., Brun, A.S., Miesch, M.S., \& Toomre, J., 2011, Astrophys. J. Lett., 739, L38

Nelson, N.J., Brown, B.P., Brun, A.S., Miesch, M.S., \& Toomre, J., 2013, Astrophys. J., 762,73

Nelson, N.J., Brown, B.P., Sacha Brun, A., Miesch, M.S., \& Toomre, J., 2014, Sol. Phys., 289,441

Nishikawa, N., \& Kusano, K., 2008, Phys. Plasmas, 15, 082903

Passos, D., Miesch, M.S., Guerrero, G., \& Charbonneau, P., 2017, Astron. Astrophys., 607, A120

Pedlosky, J., 1982, Geophys. Fluid Dyn.

Pinto, R.F., \& Brun, A.S., 2013, Astrophys. J., 772, 55

Pinto, R.F., Brun, A.S., Jouve, L., \& Grappin, R., 2011, Astrophys. J., 737, 72

Pizzolato, N., Maggio, A., Micela, G., Sciortino, S., \& Ventura, P., 2003, Astron. Astrophys. J., 397, 147

Racine, É., Charbonneau, P., Ghizaru, M., Bouchat, A., \& Smolarkiewicz, P.K., 2011, Astrophys. J., 735, 46 
Rauer, H., Catala, C., Aerts, C., et al., 2014, Exp. Astron., 38, 249

Reinhold, T., \& Gizon, L., 2015, Astron. Astrophys., 583, A65

Réville, V., Brun, A., Matt, S., Strugarek, A., \& Pinto, R., 2014, Astrophys. J., in press Réville, V., Folsom, C.P., Strugarek, A., \& Brun, A.S., 2016, Astrophys. J., 832, 145

Rieutord, M., \& Rincon, F., 2010, Living Rev. Sol. Phys., 7, 2

Rogers, T.M., 2011, Astrophys. J., 733, 12

Rubin, V.C., Burstein, D., Ford, W.K.J., \& Thonnard, N., 1985, Astrophys. J., 289, 81

Rüdiger, G., \& Kitchatinov, L.L., 1997, Astro. Nach., 318, 273

Rüdiger, G., \& Kitchatinov, L.L., 2007, NJP, 9, 302

Saar, S., 2002, Stellar Coronae in the Chandra and XMM-NEWTON Era, 277, 311

Saar, S.H., 2011, in IAU Symposium, IAU Symposium, ed. D. Prasad Choudhary \& K.G. Strassmeier, Vol. 273, 61

Saar, S.H., \& Brandenburg, A., 1999, Astrophys. J., 524, 295

Schou, J., Antia, H.M., Basu, S., et al., 1998, Astrophys. J., 505, 390

Schrinner, M., Petitdemange, L., \& Dormy, E., 2012, Astrophys. J., 752, 121

Schrinner, M., Petitdemange, L., Raynaud, R., \& Dormy, E., 2014, Astron. Astrophys., $564, \mathrm{~A} 78$

Smolarkiewicz, P.K., \& Charbonneau, P., 2013, J. Comp. Phys., 236, 608

Smolarkiewicz, P.K., \& Charbonneau, P., 2013, J. Comput. Phys., 236, 608

Spiegel, E.A., \& Zahn, J.-P., 1992, Astron. Astrophys., 265, 106

Stein, R.F., 2012, Liv. Rev. Sol. Phys., 9, 4

Strugarek, A., Beaudoin, P., Brun, A.S., et al., 2016, Adv. Space Res., 58, 1538

Strugarek, A., Beaudoin, P., Charbonneau, P., Brun, A.S., \& do Nascimento, J.D., 2017, Science, 357, 185

Strugarek, A., Brun, A.S., \& Zahn, J.-P., 2011a, Astron. Astrophys. 532, 34

Strugarek, A., Brun, A.S., \& Zahn, J.-P., 2011b, Astro. Nach., 332, 891

Sule, A., Rüdiger, G., \& Arlt, R., 2005, Astron. Astrophys., 437, 1061

Takehiro, S.-i., \& Hayashi, Y.-Y., 1999, Geophys. Astrophy. Fluid Dyn., 90, 43

Thompson, M.J., Christensen-Dalsgaard, J., Miesch, M.S., \& Toomre, J., 2003, Annu. Rev. A\&A, 41, 599

Tobias, S.M., Brummell, N.H., Clune, T.L., \& Toomre, J., 2001, Astrophys. J., 549, 1183

Tobias, S.M., Diamond, P.H., \& Hughes, D.W., 2007, Astrophys. J., 667, L113

Varela, J., Strugarek, A., \& Brun, A.S., 2016, Adv. Space Res., 58, 1507

Vasil, G.M., Lecoanet, D., Brown, B.P., Wood, T.S., \& Zweibel, E.G., 2013, Astrophys. J., 773,169

Vögler, A., \& Schüssler, M., 2007, Astron. Astrophys., 465, L43

Warnecke, J., Käpylä, P.J., Käpylä, M.J., \& Brandenburg, A., 2014, Astrophys. J. Lett., 796, L12

Warnecke, J., Käpylä, P.J., Käpylä, M.J., \& Brandenburg, A., 2016, Astrophys. J., 596, A115

Warnecke, J., Käpylä, P.J., Mantere, M.J., \& Brandenburg, A., 2013, Astrophys. J., 778,141

Watson, M., 1981, Geophys. Astrophy. Fluid Dyn., 16, 285 
Weber, M.A., Fan, Y., \& Miesch, M.S., 2011, Astrophys. J., 741, 11

Weber, M.A., Fan, Y., \& Miesch, M.S., 2013, Sol. Phys., 287, 239

Wicht, J., 2002, Physics of the Earth and Planetary Interiors, 132, 281

Wicht, J., Gastine, T., Barik, A., et al., 2017, MagIC: Fluid Dynamics in a Spherical Shell Simulator, Astrophysics Source Code Library

Wood, B.E., Müller, H.-R., Zank, G.P., \& Linsky, J.L., 2002, Astrophys. J., 574, 412

Wood, T.S., \& Brummell, N.H., 2012, Astrophys. J., 755, 99

Wood, T.S., \& Garaud, P., 2011, Astrophys. J., 738, 47

Wright, N.J., \& Drake, J.J., 2016, Nature, 535, 526

Wright, N.J., Drake, J.J., Mamajek, E.E., \& Henry, G.W., 2011, Astrophys. J., 743, 48

Yadav, R.K., Gastine, T., Christensen, U.R., \& Reiners, A., 2015, Astron. Astrophys., $573, \mathrm{~A} 68$

Yoshida, M., \& Kageyama, A., 2004, Geophys. Res. Lett., 31, 12609

Zahn, J.-P., 1983, Saas-Fee Advanced Course 13, 253

Zahn, J.-P., 1991, Astron. Astrophys., 252, 179

Zahn, J.-P., 1992, Astron. Astrophys., 265, 115

Zahn, J.-P., Talon, S., \& Matias, J., 1997, Astron. Astrophys., 322, 320 